\author{
Universidade de São Paulo \\ Instituto de Física
}

\title{
LARGURAS DE ESCAPE EM RESSONÂNCIAS GIGANTES
}

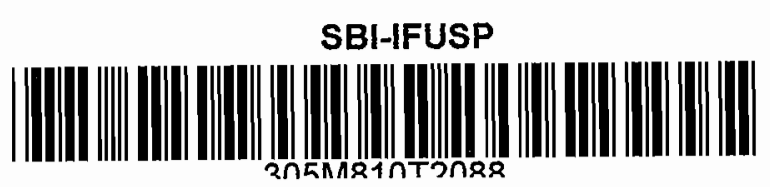

Tese de doutorado submetida ao Instituto de Física da Universidade de São Paulo

Nilton Teruya

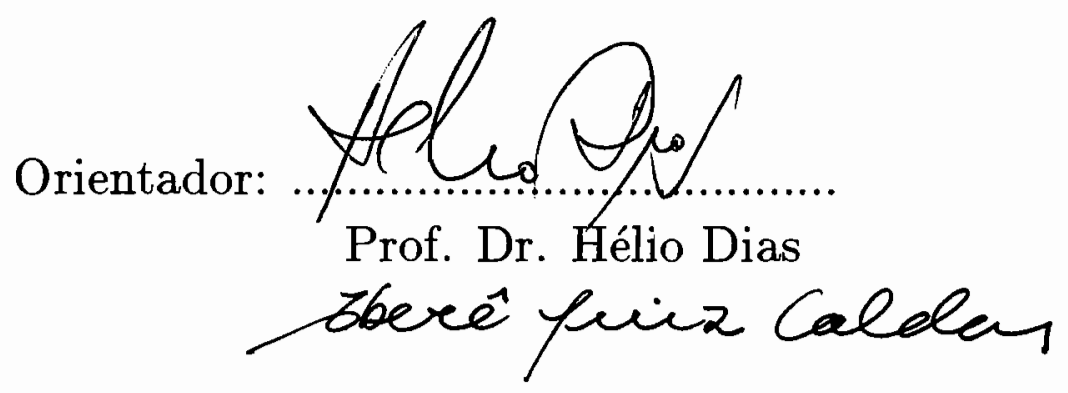

Comissão Examinadora :

Prof. Dr. HÉLIO DIAS (IFUSP)

Prof. Dr. MARCOS NOGUEIRA MARTINS (IFUSP)

Prof. Dr. CLAUDIO FABIAN TENREIRO LEIVA (IFUSP)

Prof. Dr. LUIZ FELIPE CANTO (UFRJ)

Prof. Dr. PAULO ROBERTO SILVEIRA GOMES (UFF)

São Paulo

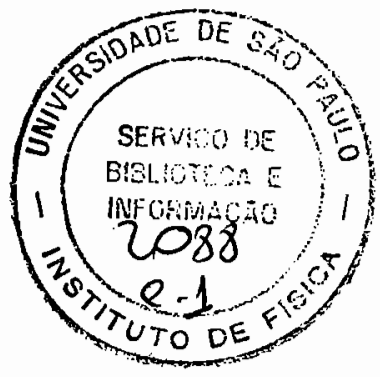




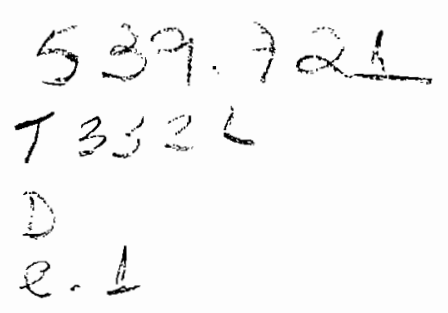

FICHA CATALOGRAFICA

Preparada pelo Serviço de Biblioteca e Informação do Instituto de Física da Universidade de são Paulo

Teruva, Nilton

Larguras de escape em resscnâncias gigantes. São Paulo, 1993.

Tese (Doutorado) - Universidade de São Paulo. Insti tuto de Física. Departamento de Física Experimental. Área de Concentração: Física Nuclear Orientador: Prof. Dr. Hélio Dias

Unitermos: 1. Ressanâncias gigantes; 2. Larguras de escape; 3. Competição entre canais.

USP/IF/SBI - 89/93 


\section{Abstract}

The energy and the escape width of the Giant Resonances are calculated utilizing the projection techniques to include the single-particle resonances in complex RPA (Random Phase Approximation) calculations. The escape widths are interpreted as the imaginary parts of the energy solution of the RPA equations. The calculations are performed for the $\operatorname{RGE} 1\left(O^{16}\right)$ and $\operatorname{RGEO}\left(Z r^{90}\right)$. For the $Z r^{90}$ the spectra of the particles ejected by the RGE0, protons and neutrons, are calculated within the hybrid model. The analysis are made through the channels independence in the hybrid model formalism. 


\section{Resumo}

As energias e larguras de escape das Ressonâncias Gigantes, são calculadas utilizando-se técnicas de projeção visando incluir as ressonâncias de partícula-única nos cálculos de RPA ("Random Phase Approximation"). As equações de RPA obtidas são complexas, e as larguras de escape são interpretadas como sendo a parte imaginária das soluções em energia. Os cálculos são feitos para a $\operatorname{RGE1}\left(O^{16}\right)$ e $\operatorname{RGE} 0\left(Z r^{90}\right)$. Para o $Z r^{90}$, os espectros das partículas emitidas pela RGE0, prótons e neutrons, são calculados através do modelo híbrido. Devido à existência de mais do que um tipo de partícula sendo emitida, as análises são feitas dentro da aproximação de independência entre os canais de partículas no formalismo do modelo híbrido. 


\section{Agradecimentos}

A realização deste trabalho de tese contou com a efetiva orientação do Prof. Hélio Dias, e com a colaboração de outros professores e colegas em várias discussões. A todos, externo meus sinceros agradecimentos. Agradeço também, às entidades de apoio à pesquisa, FAPESP e CAPES; e ainda, ao IFUSP e ao DF-UFPB pelas condições de trabalho oferecidas. 


\section{Prefácio}

O estudo da estrutura microscópica das ressonâncias gigantes (RG), tem despertado grande atenção nas pesquisas em física nuclear nos últimos anos, tendo sido desenvolvidos vários métodos de cálculos para o entendimento de suas estruturas, e também, dos mecanismos de decaimento desses modos de excitações nucleares. Além da obtenção das posições em energia, da caracterização das RG's como estados altamente coletivos, regra de soma, e do cálculo dos espectros das partículas emitidas, um tópico que tem suscitado bastante interesse, é o que diz respeito à largura desses estados. Com relação a este ponto, as análises dos espectros experimentais das partículas emitidas pelos núcleos, nestes modos de excitações, podem nos fornecer informações sobre a estrutura desses estados. Dentro deste contexto, fomos motivados a fazer um estudo detalhado da estrutura e dos modos de decaimento das RG's. No capítulo 1, discutimos a aproximação utilizada para o cálculo da energia, coletividade e largura das RG's, bem como algumas aplicações numéricas para os núcleos $O^{16}$ e $Z r^{90}$. No capítulo 2, apresentamos o formalismo utilizado para o estudo do decaimento das RG's, onde é feita uma análise para a ressonância de monopolo do $Z r^{90}$. 
O conteúdo do presente trabalho, é uma parcela de uma série de estudos que desenvolvemos sobre as RG's, parte dos quais já se encontram publicados, e outros em fase de preparação. A seguir, apresentamos a relação dos trabalhos :

- Statistical decay of the E1 giant resonance,

N.Teruya, H.Dias and E.Wolynec,

Phys.Rev.C37(1988)2121;

- E2, E4 and E6 statistical decay spectra in $P b^{208}$, N.Teruya, E.Wolynec and H.Dias, Rev.Bras.Fís.19(1989)263;

- Single particle resonances in continuum nuclear structure calculations, N.Teruya, A.F.R.de Toledo Piza and H.Dias, Phys.Rev.C44(1991)537;

- Hybrid RPA-cluster model for the dipole strength function of $L i^{11}$, N.Teruya, C.A.Bertulani, S.Krewald, H.Dias and M.S.Hussein, Phys.Rev.C43(1991)R2049;

- Calculation of giant resonance widths from damped single particle motion, Y.C.Wang, F.E.Serr, E.J.Moniz, N.Teruya, H.Dias and M.S.Hussein, Int.Jour.Mod.Phys.E1(1992)833; 
- Real solution of the complex random phase approximation equation, N.Teruya, M.Kyotoku and H.Dias, Int.Jour.Mod.Phys.E2(1993)265;

- Simple approximation to continuum random phase approximation (RPA): Application to the giant dipole resonance in $O^{16}$, N.Teruya, H.Dias and A.F.R.de Toledo Piza, Nucl.Phys.A556(1993)157;

- The channels competition in the hybrid model of the decay, N.Teruya and H.Dias, submetido ao Phys.Rev.C;

- Escape width in $Z r^{90}$, N.Teruya and H.Dias, em preparação . 


\section{Índice}

Lista de figuras $\ldots \ldots \ldots \ldots \ldots \ldots \ldots \ldots$ ix

Lista de tabelas . . . . . . . . . . . . . . . $\mathrm{x}$

Introdução 1

1 RPA no contínuo 99

$1.1 \quad \operatorname{RGE1}$ no $O^{16} \ldots \ldots \ldots \ldots \ldots \ldots \ldots \ldots \ldots \ldots \ldots$

$1.2 \quad$ RGE0 no $Z r^{90} \ldots \ldots \ldots \ldots \ldots \ldots \ldots \ldots \ldots \ldots$

2 Decaimento das ressonâncias gigantes 32

2.1 O modelo híbrido para competição entre canais . . . . . . . . . 34

2.2 Larguras de escape da RGE0 no $Z r^{90} \ldots \ldots \ldots \ldots \ldots$

2.3 Canal de prótons $(k=\pi) \ldots \ldots \ldots \ldots \ldots \ldots$

2.4 Canal de neutrons $(k=\nu) \ldots \ldots \ldots \ldots \ldots \ldots$

2.5 Cálculo do parâmetro de mistura $\mu \ldots \ldots \ldots \ldots \ldots$

3 Conclusões $\quad 56$

Apêndices : 
A Transformação da matriz complexa da RPA no contínuo em sua equivalente real

B Densidade de níveis para os núcleos $Y^{89}$ e $Z r^{89}$

C Decaimento estatístico e modelo híbrido

Referências 


\section{Lista de Figuras}

1.1 Intensidade relativa da função $S_{\Lambda}$ para a RGE1 do $O^{16} \ldots \ldots \ldots$

1.2 Porcentagem da regra da soma clássica para o $O^{16} \ldots \ldots \ldots 26$

1.3 Intensidade relativa da função $S_{\Lambda}$ para a RGE0 do $Z r^{90} \ldots \ldots . .30$

2.1 Espectro experimental de prótons para a RGE0 do $Z r^{90} \ldots \ldots \ldots 42$

2.2 Espectro experimental de neutrons para a RGE0 do $Z r^{90} \ldots \ldots .43$

2.3 Coeficientes de transmissão para prótons $\ldots \ldots \ldots \ldots$

2.4 Espectro de prótons teórico para a RGE0 do $Z r^{90} \ldots \ldots \ldots$

2.5 Coeficientes de transmissão para neutrons $\ldots \ldots \ldots \ldots \ldots$

2.6 Espectro de neutrons teórico para a RGE0 do $Z r^{9(i)} \ldots \ldots \ldots 50$ 


\section{Lista de Tabelas}

1.1 Ressonâncias de partícula-única para prótons no $O^{16} \ldots \ldots$

1.2 Parâmetros da interação residual e do potencial de Woods-Saxon para prótons $(\pi)$ e neutrons $(\nu)$ no $O^{16} \ldots \ldots \ldots \ldots$

1.3 Principais configurações $1 \mathrm{p}-1 \mathrm{~b}$ que expandem os estados $1^{-}$do $0^{16} \ldots 25$

1.4 Parâmetros da interação residual e do potencial de Woods-Saxon para prótons $(\pi)$ e neutrons $(\nu)$ no $Z r^{90} \ldots \ldots \ldots \ldots$

1.5 Principais configurações $1 \mathrm{p}-1 \mathrm{~b}$ que expandem os estados $0^{+}$do $Z r^{90}$. $\quad 29$

1.6 Larguras parciais de prótons e neutrons da RGE0 do $Z r^{90}$ (em $\mathrm{MeV}$ ). 30

2.1 Possíveis canais de saída na RGE0 do $Z r^{90}$, as probabilidades $P_{k}$ 's são valores extraídos dos resultados experimentais. . . . . . . . . . 40

2.2 População dos buracos do $Z r^{89}$ através da contribuição semi-direta. . 51

3.1 Resumo dos resultados obtidos nos cálculos para o $Z r^{90} \ldots$. . . . 58

B.1 Energias de partícula-independente para o $Y^{89}$ e $Z r^{89} \ldots \ldots$. . . 66

B.2 Niveis de energia e spin do $Z r^{89}$ obtidos teóricamente em comparação com os resultados experimentais. . . . . . . . . . . 67 
B.3 Distribuição dos níveis de energia do $Z r^{89} \ldots \ldots \ldots \ldots 68$

B.4 Distribuição dos níveis de energia do $Y^{89} \ldots \ldots \ldots \ldots$. . . . . 69 


\section{Introdução}

As ressonâncias gigantes ( $R G$ ) são modos de excitações nucleares presentes em todos os núcleos. Elas são caracterizadas como estados altamente coletivos, que esgotam grande parte da regra da soma ponderada em energia, e apresentam uma localização em energia que varia suavemente com o número de massa (A) de cada núcleo. A primeira RG observada experimentalmente foi a de dipolo, em experiências de fotoabsorção em núcleos pesados [Ba 47]. Com o desenvolvimento de novas técnicas experimentais, outras multipolaridades das RG's foram posteriormente observadas [Wo 87], tais como a de quadrupolo em experiências de espalhamento de elétrons [Pi 71a], ou de prótons [Le 72], e a de monopolo através do espalhamento de partículas $\alpha$ [Ha 77$]$.

Macroscópicamente, estes modos de excitações podem ser descritos através do modelo da gota líquida [Go 48, St 50], onde neutrons e prótons são considerados como dois tipos de fluidos incompressíveis, que se movimentam no interior nuclear.

De um ponto de vista microscópico, as RG's podem ser descritas como superposições coerentes de excitações 1 partícula-1 buraco (1p-1b), construídas 
através da ação de algum operador de multipolo $O_{\Lambda}$ sobre o estado fundamental do núcleo. A coerência dessas excitações vai caracterizar a coletividade das RG's com grandes probabilidades de transição. No modelo esquemático de Brown-Bolsterli [Br 59], para a descrição das ressonâncias dipolares, mostra-se que a interação entre os estados de $1 \mathrm{p}-1 \mathrm{~b}$, tratada com uma interação residual de dois corpos, fornece um estado coletivo localizado acima das energias não perturbadas das configurações $1 p-1 b$, carregando grande parte da intensidade de dipolo. O estado coletivo situa-se abaixo ou acima das energias não perturbadas, dependendo se a interação é atrativa (isoescalar) ou repulsiva (isovetorial) . A RPA ("Random Phase Approximation") é um dos métodos mais utilizados em cálculos microcópicos de estrutura nuclear, na descrição de estados coletivos a partir das excitações 1p-1b. Esse modelo teórico, tem sido amplamente utilizado nos cálculos das energias e regras da soma das RG's [Ro 70], fornecendo resultados bastantes satisfatórios em relação aos dados experimentais.

Tendo em vista que, energeticamente as RG's situam-se acima do limiar de emissão de partículas, elas decaem principalmente emitindo partículas, sendo o seu tempo de vida caracterizado por sua largura $\Gamma$. Em núcleos de massas médias e pesadas o decaimento por emissão de neutrons é o principal canal, já que o decaimento por emissão de prótons ou de partículas $\alpha$ é altamente inibido pela barreira coulombiana. Dessa forma, os cálculos microscópicos devem levar em conta o contínuo para que possam fornecer informações a respeito das larguras das RG's. A descrição da RG em termos das configurações de partícula-buraco, permite 
escrever a largura total $\Gamma$ em duas componentes : a largura de escape, $\Gamma^{\dagger}$, devido ao acoplamento das excitações 1p-1b ao contínuo (decaimento semi-direto), e a largura de distribuição, $\Gamma^{\downarrow}$, causada pelo acoplamento com excitações mais complicadas. Durante os estágios de evolução das excitações de partícula-buraco ( $2 \mathrm{p}-2 \mathrm{~b}$, $3 \mathrm{p}-3 \mathrm{~b}, \ldots$ ) o sistema pode decair emitindo partículas, o que é chamado de préequilíbrio, ou ir evoluindo até alcançar o equilíbrio no núcleo composto (NC), e depois decair ( decaimento estatístico ). A largura total $\Gamma$ é escrita como a soma destas componentes $\left(\Gamma=\Gamma^{\uparrow}+\Gamma^{\downarrow}\right)$.

A inclusão do contínuo em cálculos microscópicos, já foi implementada através de várias aproximações [Bu 67, Sh 75, Li 76, Ha 82, Ch 87], assim como a inclusão da fragmentação da RG em excitações de 2p-2b [De 77, Ha 82, Yo 83, Sc 84]. Recentemente, a inclusão do contínuo em cálculos de estrutura, envolvendo hamiltonianas efetivas complexas em cálculos de RPA [Gi 87, Pi 87, Cu 89], tem espertado bastante interesse, devido a possibilidade de se obter informações sobre a largura de escape da RG. Nestes cálculos, se a ressonância for isolada, a largura de escape pode ser escrita como uma soma das várias larguras parciais das excitações $1 \mathrm{p}-1 \mathrm{~b}$, nas quais a partícula está no contínuo.

Ao lado destes cálculos de estrutura, as análises dos espectros experimentais das partículas emitidas no decaimento das RG's, também podem fornecer informações a respeito das larguras $\Gamma^{\uparrow}$ e $\Gamma^{\downarrow}$, propiciando um mecanismo de comparação com os resultados previstos pelos modelos microscópicos de estrutura nuclear[Br 88, $\mathrm{Br} 89]$. 
As informações a respeito das larguras $\Gamma^{\dagger} \mathrm{e} \Gamma^{\downarrow}$, provenientes da análise dos espectros das partículas emitidas, podem ser extraídas por meio do cálculo estatístico de Hauser-Feshbach (HF)[Ha 52, Ca 69, Ey 84, Di 84, Di 86a, Br 87], ou através do modelo híbrido [Di 86b]. Nos cálculos de HF, assume-se que o decaimento ocorre através da formação do NC, e calcula-se o espectro de evaporação das partículas emitidas. Os desvios em relação ao cálculo estatístico são atribuidos à componente semi-direta do decaimento, e dessa forma avalia-se a largura de escape $\Gamma^{\uparrow}$. No modelo híbrido [Di 86b], tanto a componente semi-direta, quanto a estatística, são consideradas no cálculo do espectro das partículas emitidas, sendo que a componente estatística é normalizada a um parâmetro $\mu, \mu=\Gamma^{\downarrow} / \Gamma$, que mede o grau de fragmentação da RG para o NC ( no caso do cálculo estatístico de HF temos $\mu=1)$. As análises da RGE0 do $P b^{208}$ efetuadas com estes modelos, indicam a predominância do decaimento estatístico $(\mu \approx 1)$.

Preliminarmente a este trabalho de tese, efetuamos alguns estudos sobre os modos de decaimento das RG's, no contexto do cálculo estatístico, cujos principais resultados são suscintamente relatados a seguir. Dando continuidade aos estudos do decaimento do $P b^{208}$, iniciados com o decaimento da RGE0 (Di 84 e Di 86a), analisamos ainda, o decaimento da RGE1 [Te 88] e da RGE2 [Te 89] neste núcleo. As ressonâncias de monopolo e de dipolo no $P b^{208}$ situam-se na mesma região de energia de excitação ( $E_{x} \approx 13,5 \mathrm{MeV}$ ), sendo permitida apenas a emissão de neutrons $(\nu)$. Apesar do decaimento da RGE0 ser compatível com a previsão do cálculo estatístico, verificamos, da comparação entre o resultado teórico e os dados 
experimentais de Calarco [Ca 69], na reação $P b^{208}(\gamma, \nu) P b^{207}$, que a RGE1 admite, também, uma componente semi-direta. A densidade de níveis do núcleo residual $\left(P b^{207}\right)$ utilizada neste cálculo, foi a mesma que a empregada para o decaimento da RGE0. No caso da RGE2, a comparação com o espectro experimental dos neutrons provenientes da reação $P b^{208}\left(\alpha, \alpha^{\prime}, \nu\right) P b^{207}$ [St 81], apresenta uma subestimação na contagem do número de neutrons que populam o nível $13 / 2^{+}$do $P b^{207}$. Isto evidencia que a população deste nível pode ser explicada pelo cálculo estatístico, admitindose misturas da E2 com excitações E4 e E6, uma vez que, nos dados experimentais admite-se tal possibilidade.

A partir dos resultados apresentados por Dias e outros[Di 86b], surgiu a principal motivação deste trabalho de tese: a análise detalhada do decaimento das RG's utilizando o modelo híbrido, a qual possibilitaria o estudo da competição entre os mecanismos semi-direto e estatístico do decaimento. A fim de viabilizarmos tal projeto, iniciamos o trabalho através da implementação de uma aproximação que nos permitisse calcular as larguras de escape. O resultado desta primeira etapa, está apresentado no capítulo 1, onde as larguras de escape são calculadas com uma RPA discreta, que inclui o contínuo através das energias complexas das ressonâncias de partícula- única [Pi 87]. Por conseguinte, a matriz das equações de RPA é complexa. Como o cálculo envolve somente excitações $1 \mathrm{p}-1 \mathrm{~b}$, obtém-se apenas a largura de escape, a qual é interpretad a como sendo a parte imaginária da solução em energia das equações de RPA. Os cálculos são realizados para a RGE1 do $O^{16}$ [Te 93a] e RGE0 do $Z r^{90}$. 
As energias e larguras das ressonâncias de partícula-única, que entram nas equações de RPA, calculadas com a técnica de projeção desenvolvida por Wang-Shakin [Wa 70], apresentam valores dependentes da particular escolha de suas funções de onda. Devido a tal sensibilidade, fizemos um estudo [Te 91a] sobre a estabilidade dessas quantidades em relação à definição da função de onda, e o resultado foi implementado no código computacional TABOO [Pi 71b].

O cálculo das larguras de escape necessita então, da diagonalização de uma matriz complexa, da qual decorrem algumas dificuldades numéricas, e para superá-las, desenvolvemos um método [Te 93b] onde a matriz complexa é transformada em uma matriz real de mesma ordem, simplificando o problema para a diagonalização de uma matriz real.

Este método de inclusão do contínuo na descrição das propriedades das RG's, pode ser ampliado para se levar em conta, também, excitações de 2p2b. Dentro deste contexto, pode-se incluir aglomerados ("clusters") de partículas na base que expande as RG's. Neste sentido, paralelamente ao trabalho de tese, participamos em colaboração, no desenvolvimento de um modelo de RPA híbrida no contínuo [Te 91b], visando o cálculo da intensidade de dipolo para o $L i^{11}$. Esta RPA híbrida envolve excitações de $1 \mathrm{p}-1 \mathrm{~b}$ para neutrons e prótons, e também as excitações do aglomerado di-nêutron ( $2 \nu)$ na base de configurações . A inclusão do di-nêutron, resulta em um estado $1^{-}$no $L i^{11}$ localizado em $1,81 \mathrm{MeV}$ de energia de excitação, com uma largura de $4,0 \mathrm{MeV}$, apresentando um bom acordo com os cálculos de Sagawa e Honma [Sa 90]. 
Uma vez viabilizado o cálculo das larguras de escape, passamos a segunda etapa deste trabalho, que trata da aplicação do modelo híbrido na descrição de um sistema com mais de um canal de partícula aberto no decaimento. A escolha de um sistema com tal característica, deve-se ao fato de poder-se estudar, não só a competição entre as componentes semi-direta e estatística, como também, a competição entre os canais de diferentes tipos de partículas presentes no decaimento. Assim, apresentamos no capítulo 2, um estudo sobre o decaimento da RGE0 do $Z r^{90}$. Para este núcleo, existem três tipos de partículas passíveis de serem emitidas: prótons, neutrons e alfas. A aplicação do modelo híbrido em sistemas onde existe competição entre diversos canais de partículas, provoca certos inconvenientes nas análises, no tocante ao entendimento e controle do parâmetro de mistura $\mu$. Por exemplo, no modelo híbrido temos que o valor de $\mu$ deve ser o mesmo para cada espectro de partícula, não deixando muito transparentes as proporções em que ocorrem o decaimento estatístico e o semi-direto em cada um destes espectros.

Fizemos então, um estudo sobre a possibilidade de desmembrar o parâmetro $\mu$ nos vários canais de partícula [Te 93c]. Dessa forma, cada tipo de partícula emitida teria seu $\mu$ correspondente. A aplicação desse método ao decaimento da RGE0 do $Z r^{90}$ [Te 93d], permitiu entender de forma bastante simples, o mecanismo da competição entre os diferentes canais de partículas, e para cada um deles, a competição entre os modos de decaimento semi-direto e estatístico.

Finalmente, no capítulo 3, apresentamos um resumo dos resultados obtidos e as conclusões provenientes destes cálculos. Nos apêndices A, B e 
C detalhamos alguns pontos referentes aos cálculos dos caps.1 e 2. No apêndice A, descrevemos o método para a transformação da matriz complexa em uma real de mesma ordem; no apêndice $B$, o modelo utilizado no cálculo das densidades de níveis dos núcleos residuais resultantes do decaimento do $Z r^{90}$, e no apêndice $C$, um resumo das teorias do decaimento estatístico e do modelo híbrido. 


\section{Capítulo 1}

\section{RPA no contínuo}

A emissão de uma partícula por um núcleo excitado a uma certa energia de excitação (p.ex., na $R G$ ), pode ser entendida por meio de cálculos microscópicos de estrutura nuclear, onde o estado fundamental do núcleo é levado a um modo de excitação nuclear coletivo, no qual as excitações do tipo 1 partícula-1buraco (1p-1b) participam coerentemente. Para obtermos os efeitos de escape da partícula para o contínuo, é necessário que o contínuo seja levado em conta nestes cálculos. A implementação do contínuo nos cálculos de RPA que utilizamos, é aquela desenvolvida por Piza [Pi 87], onde a idéia básica é a inclusão dos efeitos de escape na base de configurações de $1 \mathrm{p}-1 \mathrm{~b}$ que expandem a RG. Tais efeitos são representados pelas energias complexas das configurações de $1 \mathrm{p}-1 \mathrm{~b}$, quando o estado de partícula-única pertence ao contínuo, $\hat{\varepsilon}_{p b}=\hat{\varepsilon}_{p}-\hat{\varepsilon}_{b}$, com $\varepsilon_{p}>0$, onde $\hat{\varepsilon}_{p}\left(\hat{\varepsilon}_{b}\right)$ são as energias de partícula(buraco)-única do espaço de configurações de 1p-1b, sendo a parte real $\left(\varepsilon_{p}\right)$ de $\hat{\varepsilon}_{p}$ a energia e a parte imaginária a largura da ressonância, $\Gamma_{p}$. Desta forma, obte- 
mos uma RPA discreta do mesmo modo que na RPA comumente utilizada [Ro 70], mas com energias de partícula-buraco complexas, quando a configuração de $1 \mathrm{p}-1 \mathrm{~b}$ envolver estados do contínuo.

Os estados de partícula-única do contínuo são tratados com o método desenvolvido por Wang-Shakin [Wa 70], onde a solução $\left|\psi^{+}\right\rangle$da equação de Schröedinger para o problema de espalhamento envolvendo uma hamiltoniana de campo médio, $h_{0}$, com potencial de Woods-Saxon, é projetada em dois subespaços ortogonais e complementares $\mathbf{q}$ e $\mathbf{p}$ :

$$
\left(\varepsilon-h_{0}\right)\left|\psi^{+}\right\rangle=0
$$

onde,

$$
\left|\psi^{+}\right\rangle=q\left|\psi^{+}\right\rangle+p\left|\psi^{+}\right\rangle
$$

A componente $q\left|\psi^{+}\right\rangle$tem norma finita e contém o comportamento ressonante de $\left|\psi^{+}\right\rangle$no interior nuclear, e $p=1-q$ projeta para o contínuo não ressonante (residual), o qual tem uma pequena componente dentro do núcleo. Podemos reescrever a eq.1.1, após a substituição da função de onda da eq.1.2, obtendo as seguintes equações :

$$
\begin{array}{r}
\left(\varepsilon-h_{0 q q}-h_{0 q_{1}} \frac{1}{\varepsilon-h_{0 p p}}-h_{0 p q}\right) q\left|\psi^{+}\right\rangle=h_{0 q p}\left|\psi^{+}\right\rangle, \\
\left(\varepsilon-h_{0 p p}\right)\left|\varphi^{+}\right\rangle=0,
\end{array}
$$

onde $h_{0 q q}=q h_{0} q, h_{0 q p}=q h_{0} p, q^{2}=q, p^{2}=p, q p=0 \mathrm{e}\left|\varphi^{+}\right\rangle$é a solução da hamiltoniana p! jetada $h_{0 p p}$ na energia $\varepsilon$. A partir destas equações, obtemos as 
componentes $q\left|\psi^{+}\right\rangle$e $p\left|\psi^{+}\right\rangle$, de 1.2 , nas seguintes formas:

$$
q\left|\psi^{+}\right\rangle=\frac{q h_{0}\left|\varphi^{+}\right\rangle}{\varepsilon-h_{0 q q}-h_{0 q p} \frac{1}{\varepsilon-h_{0 p p}}}
$$

e

$$
p\left|\psi^{+}\right\rangle=\left|\varphi^{+}\right\rangle+\frac{1}{\varepsilon-h_{0 p p}} h_{0 p q} q\left|\psi^{+}\right\rangle
$$

Assumindo que em alguma energia, $\varepsilon=\varepsilon_{0}>0$, o projetor $q$ projeta $\left|\psi^{+}\right\rangle$para um estado de partícula- única, $|u\rangle$, com $q=|u\rangle\langle u|$, temos que a função de onda deste estado, pode ser tomada como sendo proporcional [Wa 70] à $\left|\psi^{+}\right\rangle$na energia $\varepsilon=\varepsilon_{0}$ em que ocorre o estado ressonante. Tomamos $|u\rangle$ na energia em que $\left\langle\psi^{+} \mid \psi^{+}\right\rangle$é máximo dentro do poço de potencial, definindo dessa forma a energia $\varepsilon_{0}$ em torno da qual a ressonância deve ocorrer, resultando na seguinte função de onda :

$$
\langle r \mid u\rangle=N \frac{1}{1+e^{\left(r-R_{u}\right) / a_{u}}}\left\langle r \mid \psi^{+}\right\rangle_{\varepsilon=\varepsilon_{0}}
$$

onde $R_{u}$ e $a_{u}$ são os raio e difusibilidade para o corte em $\left|\psi^{+}\right\rangle_{\varepsilon=\varepsilon_{0}}$, e $N$ é uma constante de normalização que faz $|u\rangle$ ser normalizada a $1(\langle u \mid u\rangle=1)$.

Uma vez definidas a função de onda $|u\rangle$, e a energia $\varepsilon_{0}$, vamos completar as soluções das eqs. 1.5 e 1.6 , resolvendo a eq.1.4 para $\left|\varphi^{+}\right\rangle$, e depois vamos obter o denominador em energia da componente $q\left|\psi^{+}\right\rangle$da eq.1.5. Seguindo Wa 70 , reescrevemos a eq. 1.4 com $p=1-q$ para obtermos:

$$
\left(\varepsilon-h_{0}\right)\left|\varphi^{+}\right\rangle=\alpha|u\rangle,
$$


com $\alpha=-\left\langle u\left|h_{0}\right| \varphi^{+}\right\rangle$, sendo um parâmetro que garante a ortogonalização entre os subespaços $q$ e $p$, ou seja :

$$
\left\langle u \mid \varphi^{+}\right\rangle=0 .
$$

$O$ denominador em 1.5 é obtido definindo a quantidade $\left|\chi^{+}\right\rangle$na eq.1.5, e utilizando $q=|u\rangle\langle u|$, obtemos:

$$
\left|\chi^{+}\right\rangle=\frac{1}{\varepsilon-h_{0 p p}} h_{0}|u\rangle,
$$

a qual satisfaz, similarmente à eq.1.8 para $\left|\varphi^{+}\right\rangle$, a equação :

$$
\left(\varepsilon-h_{0}\right)\left|\chi^{+}\right\rangle=h_{0}|u\rangle-\beta|u\rangle .
$$

O parâmetro $\beta$ é obtido através da condição de contorno $\left\langle u \mid \chi^{+}\right\rangle=0$, a qual resulta na seguinte expressão para $\beta$ :

$$
\beta=\left\langle u\left|h_{0}\right| u\right\rangle+\left\langle u\left|h_{0} \frac{1}{\varepsilon-h_{o p p}} h_{0}\right| u\right\rangle .
$$

Da equação acima, podemos observar que $\beta$ tem dimensões de energia, e definindo as seguintes quantidades :

$$
\begin{aligned}
\varepsilon_{u}=\left\langle u\left|h_{0}\right| u\right\rangle, & \\
\left\langle u\left|h_{0} \frac{1}{\varepsilon-h_{0 p p}} h_{0}\right| u\right\rangle & =\left\langle u\left|h_{0} \frac{P}{\varepsilon-h_{0 p p}} h_{0}\right| u\right\rangle-i \pi\left|\left\langle u\left|h_{0}\right| \varphi^{+}\right\rangle\right|^{2} \\
& =\Delta_{u}(\varepsilon)-\frac{i}{2} \Gamma_{u}(\varepsilon),
\end{aligned}
$$

onde $P$ é o valor principal, reescrevemos $\beta$ da eq.1.12 como :

$$
\beta=\varepsilon_{u}+\Delta_{u}(\varepsilon)-\frac{i}{2} \Gamma_{u}(\varepsilon) .
$$


A obtenção do parâmetro $\beta$ vai nos fornecer a localização em energia da ressonância, assim como a sua largura, a qual é dada pela parte imaginária de $\beta$ na eq.1.14. Em vez de obtermos $\beta$ resolvendo a eq.1.11, vamos obtê-lo através das soluções das eqs.1.1 e 1.8. Primeiramente escrevemos $\left|\varphi^{+}\right\rangle$, da eq.1.8, como:

$$
\left|\varphi^{+}\right\rangle=\left|\psi^{+}\right\rangle+\alpha \frac{1}{\varepsilon-h_{0}}|u\rangle,
$$

e substituindo $\beta$ na eq.1.5, notando que $q=|u\rangle\langle u|$, temos :

$$
\begin{aligned}
\left\langle u \mid \psi^{+}\right\rangle & =\frac{\left\langle u\left|h_{0}\right| \varphi^{+}\right\rangle}{\varepsilon-\beta} \\
& =-\frac{\alpha}{\varepsilon-\beta} .
\end{aligned}
$$

Agora, utilizamos a condição $\left\langle u \mid \varphi^{+}\right\rangle=0$, da eq.1.9, e substituindo-a na eq.1.15, obtemos :

$$
\begin{aligned}
\left\langle u\left|\frac{1}{\varepsilon-h_{0}}\right| u\right\rangle & =-\frac{\left\langle u \mid \psi^{+}\right\rangle}{\alpha} \\
& =\frac{1}{\varepsilon-\beta}
\end{aligned}
$$

o que nos fornece $\beta$ através do valor esperado de $\left(\varepsilon-h_{0}\right)^{-1}$ no estado $|u\rangle$, completando, assim, as soluções das eqs.1.5 e 1.6 :

$$
\begin{aligned}
\left\langle u \mid \psi^{+}\right\rangle & =-\frac{\alpha}{\varepsilon-\beta}=-\alpha\left\langle u\left|\frac{1}{\varepsilon-h_{0}}\right| u\right\rangle \\
p\left|\psi^{+}\right\rangle & =\left|\varphi^{+}\right\rangle-\alpha\left\langle u\left|\frac{1}{\varepsilon-h_{0}}\right| u\right\rangle \frac{1}{\varepsilon-h_{0 p p}} h_{0}|u\rangle
\end{aligned}
$$

Podemos, neste ponto, escrever a matriz de transição,$T_{\varphi}$, para a hamiltoniana projetada $h_{0 p p}$, da eq.1.8, em termos da matriz $T$ da eq.1.1. $\because$ que 
fornece através da eq. 1.15 :

$$
\begin{aligned}
T_{\varphi} & =T-\left\langle\psi^{-} \mid u\right\rangle \frac{\left\langle u \mid \psi^{+}\right\rangle}{\left\langle u\left|\frac{1}{\varepsilon-h_{0}}\right| u\right\rangle} \\
& =T-S \frac{\left|\left\langle u \mid \psi^{+}\right\rangle\right|^{2}}{\left\langle u\left|\frac{1}{\varepsilon-h_{0}}\right| u\right\rangle}
\end{aligned}
$$

Substituindo as eqs. 1.16 e 1.17 na eq.1.20, temos :

$$
T_{\varphi}=T-\frac{S}{2 \pi} \frac{\Gamma_{u}(\varepsilon)}{\varepsilon-\varepsilon_{u}-\Delta_{u}(\varepsilon)-\frac{i}{2} \Gamma_{u}(\varepsilon)}
$$

fornecendo as matrizes de espalhamento $S_{\varphi}, S$ e $S^{\prime}$, com suas respectivas fases de deslocamento $\delta_{\varphi}, \delta$ e $\delta^{\prime}\left(S=e^{2 i \delta}\right)$ :

$$
\begin{aligned}
S_{\varphi} & =S \frac{\varepsilon-\varepsilon_{u}-\Delta_{u}(\varepsilon)+\frac{i}{2} \Gamma_{u}(\varepsilon)}{\varepsilon-\varepsilon_{u}-\Delta_{u}(\varepsilon)-\frac{i}{2} \Gamma_{u}(\varepsilon)} \\
e^{2 i \delta_{\varphi}} & =e^{2 i\left(\delta+\delta^{\prime}\right)}
\end{aligned}
$$

onde $\delta^{\prime}$ é uma fase que modifica a fase original de deslocamento $\delta$, dando uma matriz projetada $S_{\varphi}$, sem caráter ressonante [Wa 70]. A fase $\delta^{\prime}$ é obtida das eqs.1.22 e 1.23 :

$$
\operatorname{tg} \delta^{\prime}=\frac{\frac{1}{2} \Gamma_{u}(\varepsilon)}{\varepsilon-\varepsilon_{u}-\bar{\Delta}_{u}(\varepsilon)} .
$$

Temos, então, que a matriz de espalhamento $S$ apresenta um caráter ressonante na energia definida na eq.1.14. Desta forma, definimos as energias complexas $\hat{\varepsilon}_{R}$, dos estados de partícula-única $|u\rangle$, como :

$$
\hat{\varepsilon}_{R}=\varepsilon_{R}-\frac{i}{2} \Gamma_{R}
$$


onde $\varepsilon_{R}$ é a energia da ressonância, corrigindo a energia $\varepsilon=\varepsilon_{0}$ em que $|u\rangle$ foi definida, e $\Gamma_{R}$ é a sua largura :

$$
\begin{aligned}
\varepsilon_{R} & =\varepsilon_{u}+\Delta_{u}\left(\varepsilon_{R}\right), \\
\Gamma_{R} & =\frac{\Gamma_{u}\left(\varepsilon_{R}\right)}{1-\frac{d \Delta_{u}\left(\varepsilon_{R}\right)}{d \varepsilon}} .
\end{aligned}
$$

Para controlarmos a dependência dos deslocamentos em energia $\left(\Delta_{u}(\varepsilon)\right)$, e da largura $\left(\Gamma_{u}(\varepsilon)\right)$ em relação a uma particular escolha dos parâmetros de corte $R_{u}$ e $a_{u}$ da ressonância $|u\rangle$ (vide eq.1.7), fazemos uma expansão linear em energia para $\Delta_{u}\left(\varepsilon_{R}\right)$ e $\Gamma_{u}\left(\varepsilon_{R}\right)$ em torno de $\varepsilon=\varepsilon_{0}$, e escolhemos os parâmetros que minimizam a dependência destes parâmetros com a energia :

$$
\begin{aligned}
\varepsilon_{R} & \approx \varepsilon_{u}+\frac{\Delta_{u}\left(\varepsilon_{0}\right)+\left(\varepsilon_{u}-\varepsilon_{0}\right) \frac{d \Delta_{u}\left(\varepsilon_{0}\right)}{d \varepsilon}}{1-\frac{d \Delta_{u}\left(\varepsilon_{0}\right)}{d \varepsilon}} \\
\Gamma_{R} & \approx \frac{\Gamma_{u}\left(\varepsilon_{0}\right)+\left(\varepsilon_{R}-\varepsilon_{0}\right) \frac{d \Gamma_{u}\left(\varepsilon_{0}\right)}{d \varepsilon}}{1-\frac{d \Delta_{u}\left(\varepsilon_{0}\right)}{d \varepsilon}}
\end{aligned}
$$

A tab.1.1 mostra as ressonâncias $1 d_{3 / 2}$ e $1 f_{7 / 2}$ para prótons no $O^{16}$ para vários parâmetros de corte em $|u\rangle$.

Com esta técnica de cálculo das ressonâncias de partícula-única, podemos usar estes estados para obtermos uma base discreta de configuraçôes de $1 \mathrm{p}-1 \mathrm{~b}$, onde os estados do contínuo são representados por estas ressonâncias de energias complexas. Definindo uma hamiltoniana efetiva, $\tilde{h}_{0}$, na forma :

$$
\tilde{h}_{0}=h_{0 q q}+h_{0 q p} \frac{1}{\varepsilon-h_{0 p p}} h_{0 p q}
$$


Tabela 1.1: Ressonâncias de partícula-única, para prótons no $O^{16}$, para vários parâmetros de corte da função de onda $u(r)$, conforme eq.1.7. A linha O.H., corresponde à utilização da função de onda do oscilador harmônico $\left(\hbar \omega=41 A^{-1 / 3}\right)$.

\begin{tabular}{|c|c|c|c|c|c|c|c|}
\hline & $\begin{array}{c}R_{u} \\
(f m)\end{array}$ & $\begin{array}{c}a_{u} \\
(f m)\end{array}$ & $\left.\frac{d \Gamma_{u}}{d \varepsilon}\right|_{\varepsilon=\varepsilon_{0}}$ & $1-\left.\frac{d \Delta_{u}}{d \varepsilon}\right|_{\varepsilon=\varepsilon_{0}}$ & $\begin{array}{l}\Gamma_{u}\left(\varepsilon_{0}\right) \\
(\mathrm{MeV})\end{array}$ & $\begin{array}{c}\Gamma_{R} \\
(\mathrm{MeV})\end{array}$ & $\begin{array}{c}\varepsilon_{R} \\
(M e V)\end{array}$ \\
\hline \multirow{4}{*}{$\pi_{1 d_{3 / 2}}$} & 3,20 & 0,65 & 1,120 & 1,371 & 1,761 & 1,329 & 4,555 \\
\hline & 7,00 & 0,65 & 0,289 & 0,806 & 0,931 & 1,135 & 4,445 \\
\hline & 15,00 & 0,65 & 0,010 & 0,742 & 0,787 & 1,059 & 4,345 \\
\hline & 7,00 & 2,00 & 0,206 & 0,784 & 0,884 & 1,106 & 4,417 \\
\hline \multirow{3}{*}{$\begin{array}{c}\varepsilon_{0}=4,500 \\
(\mathrm{MeV})\end{array}$} & 10,00 & 2,00 & 0,092 & 0,753 & 0,821 & 1,075 & 4,378 \\
\hline & 15,00 & 2,00 & 0,020 & 0,743 & 0,791 & 1,061 & 4,349 \\
\hline & \multicolumn{2}{|c|}{ O.H. } & 0,813 & 1,129 & 1,418 & 1,280 & 4,534 \\
\hline \multirow[t]{2}{*}{$\pi_{1 f_{7 / 2}}$} & 3,20 & 0,65 & 1,375 & 1,207 & 5,193 & 4,899 & 11,134 \\
\hline & 7,00 & 2,00 & 0,136 & 0,722 & 2,429 & 3,311 & 10,330 \\
\hline \multirow{3}{*}{$\begin{array}{c}\varepsilon_{0}=10,610 \\
(\mathrm{MeV})\end{array}$} & 10,00 & 2,00 & 0,049 & 0,709 & 2,310 & 3,231 & 10,221 \\
\hline & 15,00 & 2,00 & 0,012 & 0,706 & 2,265 & 3,201 & 10,171 \\
\hline & \multicolumn{2}{|c|}{ O.H. } & 0,626 & 0,870 & 3,356 & 3,995 & 10,802 \\
\hline
\end{tabular}


temos, das eqs.1.12 e 1.14, que o estado de partícula-única $|u\rangle$, satisfaz a equação:

$$
\left(\hat{\varepsilon}_{R}-\tilde{h}_{0}\right)|u\rangle=0 .
$$

A projeção do espaço de partícula-única nos subespaços $q$ e $p$, nos permite formar uma base de configurações de $1 \mathrm{p}-1 \mathrm{~b}$ projetada em dois subespaços $Q$ e $P$, ortogonais e complementares, onde as configurações de $1 \mathrm{p}-1 \mathrm{~b}$, tanto do contínuo $\left(\varepsilon_{p}>0\right)$, quanto dos estados ligados $\left(\varepsilon_{p}<0\right.$, neste caso $\left.\Gamma_{p}=0\right)$, expandem o sub-espaço $Q$, sendo que $P$ projeta para o contínuo residual $(p)$. Se $|\nu\rangle$ é uma solução da hamiltoniana nuclear $\left(H=H_{0}+V\right)$ :

$$
\left(E_{\nu}-H\right)|\nu\rangle=0,
$$

onde $H_{0}$ é a hamiltoniana de campo médio, e $V$ a interação residual de dois corpos, temos que a projeção nestes sub-espaços fornecem :

$$
\begin{aligned}
&\left(E_{\nu}-H_{Q Q}\right) Q|\nu\rangle=H_{Q P} P|\nu\rangle, \\
&\left(E_{\nu}-H_{P P}\right) P|\nu\rangle=H_{P Q} Q|\nu\rangle, \\
&\left(H_{Q Q}=Q H Q, H_{Q P}=Q H P, \ldots\right) .
\end{aligned}
$$

Levando em conta que as soluções de $h_{0 p p}$ no espaço de partículaúnica contribuem muito pouco no interior nuclear, vamos fazer a aproximação de que os efeitos da interação residual em $H_{Q P}, H_{P Q}$ e $H_{P P}$ possam ser desprezados, obtendo:

$$
\begin{aligned}
\left(E_{\nu}-\tilde{H}_{0}-V\right) Q|\nu\rangle & =H_{0 Q P}\left|\Phi_{c}^{+}\right\rangle \\
\left(E_{\nu}-H_{0 P P}\right)\left|\Phi_{c}^{+}\right\rangle & =0
\end{aligned}
$$


onde $\left|\Phi_{c}^{+}\right\rangle$é a solução de $H_{0 P P}$ no canal $c$, e $\tilde{H}_{0}$ é dada por :

$$
\tilde{H}_{0}=H_{0 Q Q}+H_{0 Q P} \frac{1}{E-\bar{H}_{0 P P}}-H_{0 P Q},
$$

a qual leva em conta o contínuo no segundo termo do lado direito. No espaço discreto de configurações de $1 \mathrm{p}-1 \mathrm{~b}$, a componente $Q|\nu\rangle$ pode ser escrita em termos de um grupo de estados biortogonais $\left|\tilde{R}_{n}\right\rangle$ e $\left|R_{n}\right\rangle$ que satisfazem a hamiltoniana efetiva $\tilde{H}_{Q Q}:$

$$
\begin{gathered}
\tilde{H}_{Q Q}=\tilde{H}_{0}+V, \\
\left(\hat{E}_{n}-\tilde{H}_{Q Q}\right)\left|R_{n}\right\rangle=0, \\
\left(\hat{E}_{n}^{*}-\tilde{H}_{Q Q}^{\dagger}\right)\left|\tilde{R}_{n}\right\rangle=0,
\end{gathered}
$$

com a normalização $\left\langle\tilde{R}_{n^{\prime}} \mid R_{n}\right\rangle=\delta_{n n^{\prime}}$. As energias $\hat{E}_{n}$, dos modos $\left|R_{n}\right\rangle$, são complexas, e a parte imaginária é interpretada como sendo a largura desses modos de excitação. Como já estamos particularizando o espaço que expande $Q|\nu\rangle$ com configurações de $1 \mathrm{p}-1 \mathrm{~b}$, a largura obtida é a largura de escape $\left(\Gamma_{n}^{\uparrow}\right)$. Em termos dos modos $\left|R_{n}\right\rangle$ a componente $Q|\nu\rangle$ é escrita como:

$$
Q|\nu\rangle=\sum_{n} \frac{\left|R_{n}\right\rangle\left\langle\tilde{R}_{n}\left|H_{0}\right| \Phi_{c}^{+}\right\rangle}{E_{\nu}-\hat{E}_{n}}
$$

a qual apresenta pólos no espectro discreto dos modos complexos $\left|R_{n}\right\rangle$, com amplitude de escape $\left\langle\tilde{R}_{n}\left|H_{0}\right| \Phi_{c}^{+}\right\rangle$.

Da mesma forma que na RPA comumente utilizada, podemos obter os modos $\left|R_{n}\right\rangle$ (e seu conjugado $\left|\tilde{R}_{n}\right\rangle$ ) pela ação do operador de excitação $\Omega_{n}^{\dagger}\left(\tilde{\Omega}_{n}^{\dagger}\right)$ 
sobre o estado fundamental $|0\rangle$ :

$$
\begin{aligned}
\Omega_{n}|0\rangle & =0 \\
\tilde{\Omega}_{n}|0\rangle & =0 \\
\left|R_{n}\right\rangle & =\Omega_{n}^{\dagger}|0\rangle \\
\left|\tilde{R}_{n}\right\rangle & =\tilde{\Omega}_{n}^{\dagger}|0\rangle \\
\Omega_{n}^{\dagger} & =\sum_{p b}\left(X_{p b}^{n} a_{p}^{\dagger} a_{b}-Y_{p b}^{n} a_{b}^{\dagger} a_{p}\right), \\
\tilde{\Omega}_{n}^{\dagger} & =\sum_{p b}\left(X_{p b}^{n^{*}} a_{p}^{\dagger} a_{b}-Y_{p b}^{n^{*}} a_{b}^{\dagger} a_{p}\right),
\end{aligned}
$$

onde $a^{\dagger}$ e $a$ são os operadores de criação e aniquilação de férmions, fornecendo a equação de movimento:

$$
\left\langle 0\left|\left[\tilde{\Omega}_{n},\left[\tilde{H}_{Q Q}, \Omega_{n^{\prime}}^{\dagger}\right]\right]\right| 0\right\rangle=\hat{E}_{n}\left\langle 0\left|\left[\tilde{\Omega}_{n}, \Omega_{n^{\prime}}^{\dagger}\right]\right| 0\right\rangle
$$

$\operatorname{com}\left\langle 0\left|\left[\tilde{\Omega}_{n}, \Omega_{n^{\prime}}^{\dagger}\right]\right| 0\right\rangle=\left\langle\tilde{R}_{n} \mid R_{n^{\prime}}\right\rangle=\delta_{n n^{\prime}}$ que pode ser escrita em termos das matrizes $A$ e $B$ :

$$
\left(\begin{array}{cc}
A & B \\
-B & -A
\end{array}\right)\left(\begin{array}{l}
X \\
Y
\end{array}\right)=\hat{E}\left(\begin{array}{l}
X \\
Y
\end{array}\right)
$$

onde:

$$
A_{p b p^{\prime} b^{\prime}}=\left(\varepsilon_{p^{\prime}}-\varepsilon_{b^{\prime}}\right) \delta_{p p^{\prime}} \delta_{b b^{\prime}}+\left\langle 0\left|\left[a_{b}^{\dagger} a_{p},\left[V, a_{{p^{\prime}}^{\prime}}^{\dagger} a_{b^{\prime}}\right]\right]\right| 0\right\rangle
$$

$\mathrm{e}$

$B_{p b p^{\prime} b^{\prime}}=-\left\langle 0\left|\left[a_{b}^{\dagger} a_{p},\left[V, a_{b^{\prime}}^{\dagger} a_{p^{\prime}}\right]\right]\right| 0\right\rangle$.

A diferença em relação à RPA usual, é que agora as energias de partícula-única do contínuo $\left(\varepsilon_{p^{\prime}}>0\right)$ são as energias complexas definidas na eq.1.31, 
fazendo com que os modos complexos $\left|R_{n}\right\rangle$ tenham a norma :

$$
\left\langle\tilde{R}_{n^{\prime}} \mid R_{n}\right\rangle=\delta_{n n^{\prime}}=X^{T} X-Y^{T} Y,
$$

com $X^{T}\left(Y^{T}\right)$ sendo a transposta de $X(Y)$.

A resposta do núcleo a algum operador de multipolo elétrico, $O_{\Lambda}=$ $r^{\Lambda} Y_{\Lambda}\left(O_{\Lambda=0}=r^{2}\right)$, é caracterizada pela função intensidade $S_{\Lambda}(E)$, a qual é calculada com a aproximação de que a excitação $|\nu\rangle$ possa ser bem representada por sua componente $Q|\nu\rangle$ :

$$
S_{\Lambda}(E)=\frac{1}{2 \pi} \sum_{n}\left|\left\langle\tilde{R}_{n}\left|O_{\Lambda}\right| 0\right\rangle\right|^{2} \frac{\Gamma_{n}^{\uparrow}}{\left(E-E_{n}\right)^{2}+\frac{\Gamma_{n}^{\dagger 2}}{4}}
$$

sendo $\Gamma_{n}^{\uparrow}=2 \pi\left|\left\langle\tilde{R}_{n}\left|H_{0}\right| \Phi_{c}^{+}\right\rangle\right|^{2}$, que fornece a regra da soma ponderada em energia (EWSR) num intervalo de energia de excitação variando de $E=0 \mathrm{MeV}$ à $E=E_{M}$ :

$$
E W S R=\int_{0}^{E_{M}} E S_{\Lambda}(E) d E .
$$

A solução das equações 1.41 envolve a diagonalização de uma matriz complexa de ordem $2 \mathrm{~N} \times 2 \mathrm{~N}$, onde $\mathrm{N}$ é o número de configurações de $1 \mathrm{p}-1 \mathrm{~b}$ que expandem um particular modo de excitação nuclear de momento angular e paridade $J^{\pi}\left(\vec{J}=\vec{\jmath}_{p}+\vec{\jmath}_{b} ; \pi=(-)^{l_{p}+l_{b}}\right)$.Algumas dificuldades técnicas surgem quando diagonalizamos a matriz em 1.41, as quais se apresentam, principalmente, na precisão da norma $\left\langle\tilde{R}_{n^{\prime}} \mid R_{n}\right\rangle$ em 1.42. Por exemplo, utilizando a rotina EIGCC, do pacote de subrotinas IMSL, podemos obter $\left\langle\tilde{R}_{n^{\prime}} \mid R_{n}\right\rangle$ com precisão da ordem de $10^{-1}$, o que não é um resultado muito bom. No apêndice A apresentamos um método para contornarmos este problema de precisão das soluções, o qual se baseia na transformação da matriz complexa de 1.41 em uma matriz real de mesma ordem. 
A diagonalização da hamiltoniana efetiva $\tilde{H}_{Q Q}$ no subespaço $Q$ de configurações $1 \mathrm{p}$ - 1b , foi feita utilizando uma interação residual $V$ de LandauMigdal [Ha 82] :

$$
V_{p b}\left(r, r^{\prime}\right)=C_{0}\left[f(r)+f^{\prime}(r) \vec{\tau} \cdot \vec{\tau}^{\prime}+\vec{\sigma} \cdot \vec{\sigma}^{\prime}\left(g(r)+g^{\prime}(r) \vec{\tau} \cdot \vec{\tau}^{\prime}\right)\right] \delta\left(\vec{r}-\vec{r}^{\prime}\right)
$$

na qual os parâmetros $f, f^{\prime}, g$ e $g^{\prime}$, são adimensionais e dependem da densidade $\rho(r):$

$$
F(r)=F^{e x}+\left(F^{i n}-F^{e x}\right) \rho(r),
$$

com $\mathrm{F}$ sendo qualquer um dos parâmetros $f, f^{\prime}, g$ e $g^{\prime}$ externo(ex) ou interno(in) :

$$
\rho(r)=\frac{1}{1+e^{(r-R) / a}}
$$

sendo $R$ e $a$ o raio e a difusibilidade nucleares, e $C_{0}$ uma constante de ajuste com dimensões de $M e V \cdot f m^{3}$. O elemento de matriz da interação residual em $A_{p b p^{\prime} b^{\prime}}$ de 1.41 , é dado por : 


$$
\begin{aligned}
& V_{p b p^{\prime} b^{\prime}}^{J}=\frac{C_{0}}{4 \pi}(-)^{k_{p}+k_{p^{\prime}}} \sqrt{k_{p} k_{b} \overline{k_{p^{\prime}} k_{b^{\prime}}}} \int_{0}^{\infty} R_{p}(r) R_{b}(r) R_{p^{\prime}}(r) R_{b^{\prime}}(r) r^{2} d r \\
& \left\{\left(\begin{array}{ccc}
j_{p} & j_{b} & J \\
1 / 2 & -1 / 2 & 0
\end{array}\right)\left(\begin{array}{ccc}
j_{p^{\prime}} & j_{b^{\prime}} & J \\
1 / 2 & -1 / 2 & 0
\end{array}\right) h(r)\right. \\
& \left.+(-)^{k_{b}+k_{b^{\prime}}+l_{b}+l_{b^{\prime}}}\left(\begin{array}{ccc}
j_{p} & j_{b} & J \\
1 / 2 & 1 / 2 & -1
\end{array}\right)\left(\begin{array}{ccc}
j_{p^{\prime}} & j_{b^{\prime}} & J \\
1 / 2 & 1 / 2 & -1
\end{array}\right) h^{\prime}(r)\right\} \\
& B_{p b p^{\prime} b^{\prime}}^{J}=(--)^{j_{p^{\prime}}-j_{b^{\prime}}-J} V_{p b b^{\prime} p^{\prime}}^{J}
\end{aligned}
$$

onde:

$$
\begin{aligned}
k_{i} & =j_{i}+\frac{1}{2} \\
R_{i}(r) & =\text { funções de onda radiais } \\
\vec{J} & =\vec{j}_{p}+\vec{j}_{b} \\
h(r) & =2\left[\left(f+\tau_{3} \tau_{3}^{\prime} f^{\prime}+g+\tau_{3} \tau_{3}^{\prime} g^{\prime}\right)+(-)^{l_{p}+l_{b}+J}\left(f-g+\tau_{3} \tau_{3}^{\prime}\left(f^{\prime}-g^{\prime}\right)\right)\right] \\
h^{\prime}(r) & =4\left(g+\tau_{3} \tau_{3}^{\prime} g^{\prime}\right)
\end{aligned}
$$




$$
\tau_{3} \tau_{3}^{\prime}= \begin{cases}+1 & \text { para próton-próton e nêutron-nêutron } \\ -1 & \text { para próton-nêutron e nêutron-próton }\end{cases}
$$

A aproximação discutida foi aplicada nas excitações de dipolo do $O^{16}$ e monopolo do $Z r^{90}$, as quais apresentaremos a seguir.

\subsection{RGE1 no $O^{16}$}

Os parâmetros da interação residual são aqueles utilizados por de Haro[Ha 82], exceto por uma pequena diferença na constante $C_{0}$, a qual foi obtida â. istando o estado $3^{-}$de mais baixa energia $\left(E_{3^{-}}=6,16 \mathrm{MeV}\right)$, próxima da energia experimental $\left(E_{3^{-}}=6,13 \mathrm{MeV}\right)[\mathrm{Se} 86]$, e a solução espúria de dipolo isoescalar próxima de zero $\mathrm{MeV}$. Obtivemos $C_{0}=415 \mathrm{MeV} . \mathrm{fm}^{3}$, o que é aproximadamente igual a constante de $\mathrm{Ha} 82\left(C_{0}=418,852 \mathrm{MeV} \cdot \mathrm{fm}^{3}\right)$. A hamiltoniana efetiva $\tilde{H}_{Q Q}$ foi diagonalizada numa base de oscilador harmônico $\left(\hbar \omega=41 A^{-1 / 3}\right)$ incluindo os estados de partícula-única até a onda $3 s_{1 / 2}$. Na tab.1.2 apresentamos os parâmetros da interação residual e o potencial de Woods-Saxon utilizados no cálculo, e na tab.1.3 estão as energias dos principais estados da base de configurações de 1p-1b. A parametrização do potencial de Woods-Saxon foi obtida através do aju: das energias e larguras dos estados de partícula-única $1 d_{3 / 2}$, para prótons 
Tabela 1.2: Parâmetros da interação residual e do potencial de Woods-Saxon para prótons $(\pi)$ e neutrons $(\nu)$ no $O^{16}$.

\begin{tabular}{|c|c|c|c|c|}
\hline$C_{0}\left(\mathrm{MeVfm}^{3}\right)$ & $f_{0 i n}$ & $f_{0 e x}$ & $f_{\text {Oin }}^{\prime}$ & $f_{\text {Oex }}^{\prime}$ \\
\hline 415 & 0,2 & $-2,45$ & 1,5 & 1,5 \\
\hline & $g_{0}$ & $g_{0}^{\prime}$ & $R(f m)$ & $a(f m)$ \\
\cline { 2 - 5 } & 0,55 & 0,7 & 5,2 & 0,5 \\
\hline \multicolumn{5}{|c|}{$U(r)=V_{0} f(r)+\frac{l . \sigma}{\tau} V_{l \sigma} f^{\prime}(r)$} \\
\hline particula & $V_{0}(\mathrm{MeV})$ & $V_{l \sigma}(\mathrm{MeV} f m)$ & $R_{w s}(f m)$ & $a_{w s}(f m)$ \\
\hline$\pi$ & 51,8 & 13,00 & 3,20 & 0,65 \\
\hline$\nu$ & 52,5 & 13,08 & 3,20 & 0,53 \\
\hline
\end{tabular}

$\left(\hat{\varepsilon}_{1 d_{3 / 2}}=4,379-i 0,530 \mathrm{MeV}\right)$ e neutrons $\left(\hat{\varepsilon}_{1 d_{3 / 2}}=0,892-i 0,036 \mathrm{MeV}\right)$, próximas aos dados experimentais [Se 86].

A fig.1.1 mostra a intensidade relativa de $S_{\Lambda}(E)$, onde as principais contribuições correspondem às soluções de $\tilde{H}_{Q Q}$ em $\hat{E}_{n}=17,01-i 0,45 ; 24,17$ $i 0,72$ e $25,40-i 0,42 \mathrm{MeV}$, as quais estão em bom acordo com os resultados de $\mathrm{Ha} 82$ (dois picos principais em 24,9 e $26,2 \mathrm{MeV}$ ). Em relação ao resultado experimental $\left(22,15 \mathrm{MeV}\right.$ e $\left.\Gamma^{\dagger}=1,6 \mathrm{MeV}\right)$, o nosso resultado (e o de Ha 82) está deslocado em $\sim 2,0 \mathrm{MeV}$ para cima na escala de energia, apresentando um bom 
Tabela 1.3: Principais configurações $1 \mathrm{p}-1 \mathrm{~b}$ que expandem os estados $1^{-}$do $0^{16}$.

\begin{tabular}{|c|c|c|}
\hline \multirow{2}{*}{ Configuração } & \multicolumn{2}{|c|}{$\hat{\varepsilon}_{p b}=-i \Gamma_{p b} / 2(\mathrm{MeV})$} \\
\cline { 2 - 3 } & $\pi$ & $\nu$ \\
\hline $1 p_{1 / 2}^{-1} 2 s_{1 / 2}$ & 11,887 & 13,790 \\
\hline $1 p_{3 / 2}^{-1} 2 d_{5 / 2}$ & 14,028 & 13,790 \\
\hline $1 p_{3 / 2}^{-1} 2 s_{1 / 2}$ & 14,386 & 16,351 \\
\hline $1 p_{1 / 2}^{-1} 2 d_{3 / 2}$ & $16,581-\mathrm{i} 0,530$ & $17,520-\mathrm{i} 0,036$ \\
\hline $1 p_{3 / 2}^{-1} 2 d_{3 / 2}$ & $19,080-\mathrm{i} 0,530$ & $20,081-\mathrm{i} 0,036$ \\
\hline $1 s_{1 / 2}^{-1} 2 p_{3 / 2}$ & $43,296-\mathrm{i} 12,260$ & $48,202-\mathrm{i} 15,180$ \\
\hline
\end{tabular}

acordo para a largura da ressonância. A função intensidade $S_{\Lambda}(E)$ exaure cerca de $91 \%$ da regra da soma clássica de dipolo isovetorial $\left(\sim 14,8 \frac{\mathrm{NZ}}{\mathrm{A}}\right)$ no intervalo de energia entre 0 e $E_{M}=30 \mathrm{MeV}$ (fig.1.2). 


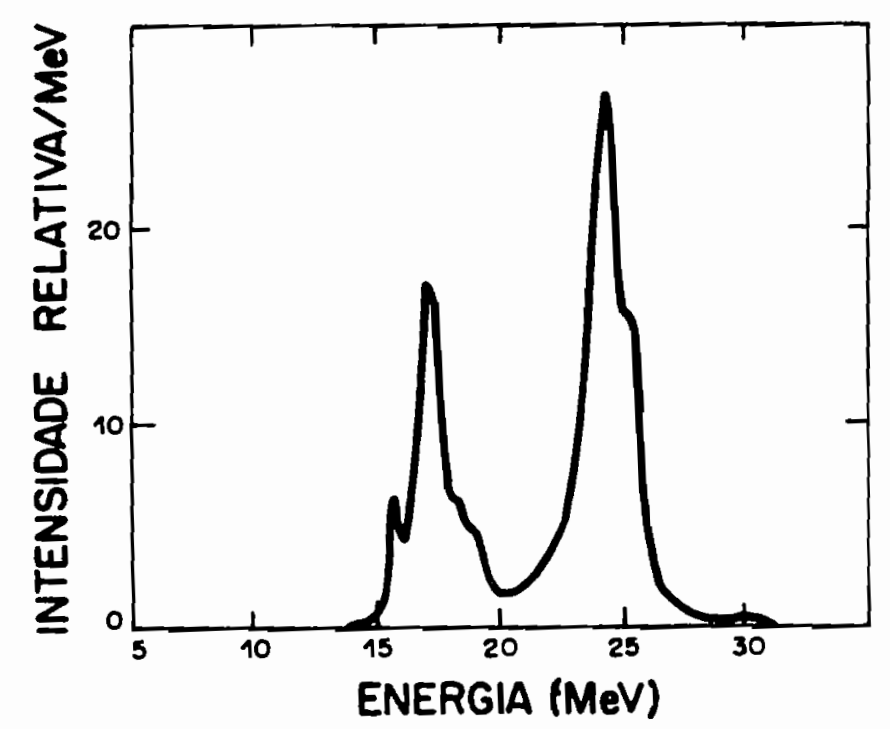

Figura 1.1: Intensidade relativa da função $S_{\Lambda}$ para a RGE1 do $O^{16}$.

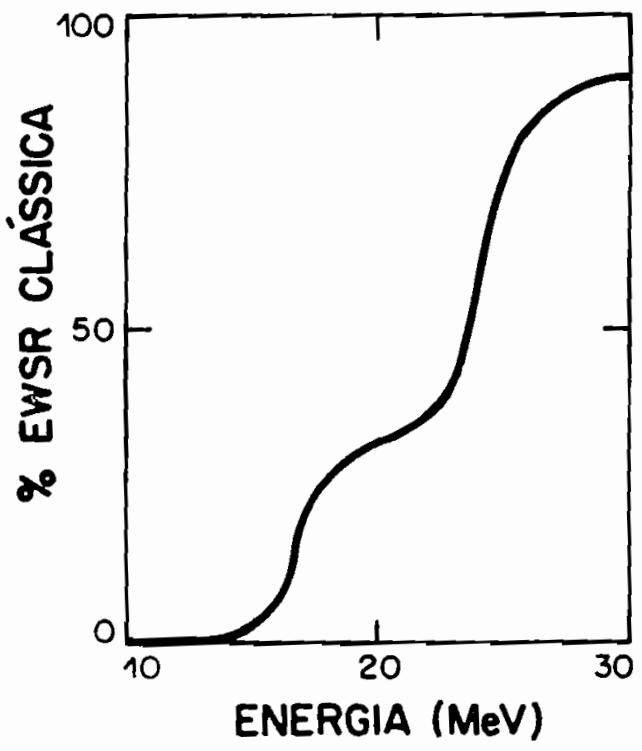

Figura 1.2: Porcentagem da regra da soma clássica para o $O^{16}$. 


\subsection{RGE0 no $Z r^{90}$}

Antes de começarmos a discussão sobre o $Z r^{90}$, vamos fazer algumas considerações pertinentes aos cálculos do $O^{16}$ da seção anterior.

Os parâmetros da interação residual utilizados no cálculo da RGE1 do $O^{16}$ (tab.1.2), fornecem um deslocamento do centróide de energia de $\sim 2,0 \mathrm{MeV}$ em relação à energia experimental. Este deslocamento também ocorre nos trabalhos de de Haro [Ha 82] e Co [Co 85], onde a parametrização da interação residual é a mesma que a utilizada neste trabalho. Tais parâmetros foram obtidos através do ajuste das energias das ressonâncias E0 e E2 (isoescalar) e E1 (isovetorial) do $P b^{208}$ no trabalho de Rinker-Speth [Ri 78]. O deslocamento de $\sim 2,0 \mathrm{MeV}$ na energia da RGE1 do $O^{16}$, deve-se principalmente à parametrização de $f_{0 i n}^{\prime}=1,5$, como apontado por de Haro [Ha 82] e Co [Co 85]. Embora $f_{0 i n}^{\prime}=1,5$ ajuste a energia da RGE1 do $P b^{208}$ [Ri 78], ele fornece uma energia de simetria $(\beta)$, da fórmula de massa de Bethe-Weizacker [Bo 69], da ordem de $53 \mathrm{MeV}$ [Br 79, Co 85] que é cerca de duas vezes o valor aceito na literatura $(\beta \approx 28-36 \mathrm{MeV})$ [ $\mathrm{Br} 79]$ :

$$
\beta=\frac{1}{3} \varepsilon_{F}\left(1+2 f_{0 i n}^{\prime}\right)
$$

sendo $\varepsilon_{F}$ a energia de Fermi $(\approx 40 \mathrm{MeV})$.

Tomando por base as discussões acima, ajustamos o parâmetro $f_{\text {oin }}^{\prime}=0,6$ para fornecer a energia de simetria $\beta \approx 28 \mathrm{MeV}$, com uma constante $C_{0}=300 \mathrm{MeV} \cdot \mathrm{fm}^{3}$ ajustada em $\varepsilon_{F} \approx 39 \mathrm{MeV}\left(C_{0}=\frac{\hbar^{2} \pi^{2}}{m^{*} k_{F}}, k_{F} \approx 1,36 \mathrm{fm}^{-1} \mathrm{e}\right.$ $\left.\frac{m^{*}}{m}=1\right)$. Com estes valores de $C_{0}$ e $f_{0 i n}^{\prime}$, ajustamos o parâmetro $f_{\text {oin }}=-0,3$ 
Tabela 1.4: Parâmetros da interação residual e do potencial de Woods-Saxon para prótons $(\pi)$ e neutrons $(\nu)$ no $Z r^{90}$.

\begin{tabular}{|c|c|c|c|c|}
\hline$C_{0}\left(M e V f m^{3}\right)$ & $f_{0 i n}$ & $f_{0 e x}$ & $f_{\text {Oin }}^{\prime}$ & $f_{0 e x}^{\prime}$ \\
\hline \multirow[t]{3}{*}{300} & $-0,3$ & $-2,45$ & 0,60 & 1,5 \\
\hline & $g_{0}$ & $g_{0}^{\prime}$ & $R(f m)$ & $a(f m)$ \\
\hline & 0,55 & 0,7 & 5,1 & 0,6 \\
\hline \multicolumn{5}{|c|}{$U(r)=V_{0} f(r)+\frac{l \cdot \sigma}{\tau} V_{l \sigma} f^{\prime}(r)$} \\
\hline particula & $V_{0}(\mathrm{MeV})$ & $V_{l \sigma}(M e V f m)$ & $R_{w s}(f m)$ & $a_{w s}(f m)$ \\
\hline$\pi$ & 55,3 & 25,5 & 5,69 & 0,70 \\
\hline$\nu$ & 48,7 & 25,5 & 5,69 & 0,70 \\
\hline
\end{tabular}

para fornecer o centróide da RGE0 do $Z r^{90}(=15,84 \mathrm{MeV})$ próxima à energia experimental $E_{x}=(16,1 \pm 0,4) \mathrm{MeV}[\mathrm{Bo} 89]$.

Na tab.1.4 apresentamos os parâmetros da interação residual e do potencial de Woods-Saxon para o $Z r^{90}$, e na tab.1.5 estão as energias das con:figurações $1 p-1 b$.

Experimentalmente [Bo 89] a RGE0 do $Z r^{90}$ está situada em 16, $1 \pm$ $0,4 \mathrm{MeV}$ com uma largura $\Gamma=3,1 \pm 0,4 \mathrm{MeV}$, esgotando $48 \%$ da EWSR clássica $\left(\frac{\hbar^{2} Z^{2}}{2 m A}\left\langle r^{2}\right\rangle\right)$. Nossos resultados são apresentados na fig.1.3, onde a ressonância está 
Tabela 1.5: Principais configurações $1 \mathrm{p}-1 \mathrm{~b}$ que expandem os estados $0^{+}$do $Z r^{90}$, as energias dos buracos são experimentais (apêndice B).

\begin{tabular}{|c|c|c|}
\hline \multirow{2}{*}{ Configuração } & \multicolumn{2}{|c|}{$\hat{\varepsilon}_{p b}=\varepsilon_{p b}-i \Gamma_{p b} / 2(M e V)$} \\
\cline { 2 - 3 } & $\nu$ & $\pi$ \\
\hline $1 g_{9 / 2}^{-1} 2 g_{9 / 2}$ & $17,10-\mathrm{i} 0,64$ & - \\
\hline $1 f_{5 / 2}^{-1} 2 f_{5 / 2}$ & $16,50-\mathrm{i} 0,43$ & $20,18-\mathrm{i} 0,94$ \\
\hline $2 p_{1 / 2}^{-1} 3 p_{1 / 2}$ & $13,10-\mathrm{i} 0,19$ & $16,21-\mathrm{i} 1,34$ \\
\hline $2 p_{3 / 2}^{-1} 3 p_{3 / 2}$ & 13,08 & $16,41-\mathrm{i} 0,62$ \\
\hline $1 f_{7 / 2}^{-1} 2 f_{7 / 2}$ & 15,15 & $20,12-\mathrm{i} 0,04$ \\
\hline
\end{tabular}

situada em $15,84 \mathrm{MeV}$, com uma largura de escape $\Gamma^{\uparrow}=0,52 \mathrm{MeV}$, esgotando $50 \%$ da EWSR clássica no intervalo entre $0-E_{M}=30 \mathrm{MeV}$ (vide eq.1.44).

As larguras $\Gamma_{n}^{\dagger}$ da eq.1.43, obtidas pela diagonalização das eqs.1.41, podem ser decompostas em termos das larguras $\Gamma_{p b}$ (tab.1.5) das configurações 1p1b. Levando em conta a norma $\left\langle\tilde{R}_{n^{\prime}} \mid R_{n}\right\rangle$ da eq.1.42, obtemos para a largura de escape :

$$
\Gamma_{n}^{\uparrow}=\sum_{p}\left\langle\tilde{R}_{n p b} \mid R_{n p b}\right\rangle \Gamma_{p b}
$$

$\left(\left|R_{n p b}\right\rangle\right.$ são as componentes $X_{p b}^{n}\left(Y_{p b}^{n}\right)$ do vetor $\left.\left|R_{n}\right\rangle\right)$. Como em nossa base de 1p-1b temos excitações de prótons e de neutrons, podemos então, escrever a largura de escape como sendo uma soma das larguras devido às excitações de prótons e de 


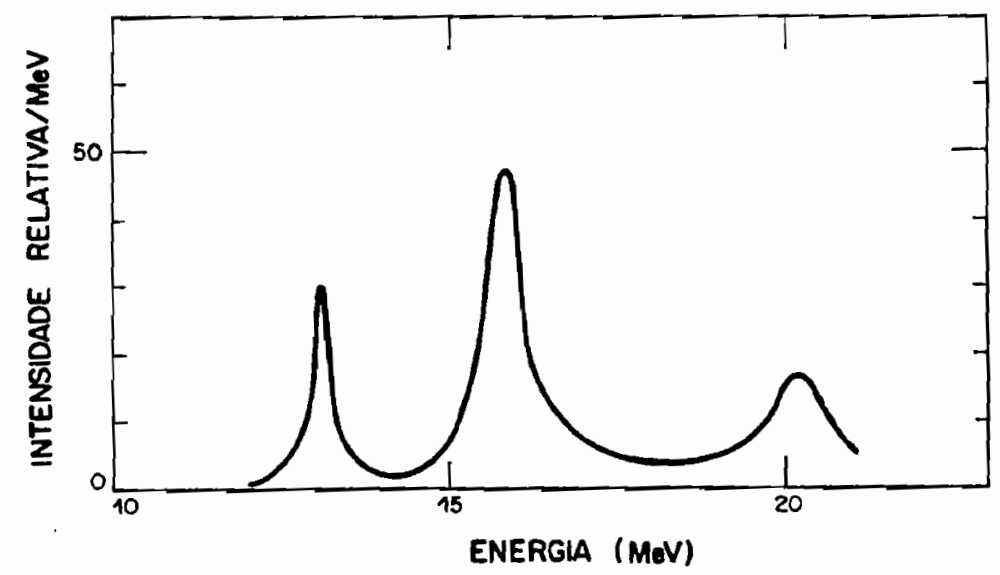

Figura 1.3: Intensidade relativa da funçầo $S_{\Lambda}$ para a RGE0 do $Z r^{90}$.

Tabela 1.6: Larguras parciais de prótons e neutrons da RGE0 do $Z r^{90}(\mathrm{em} M e V)$.

\begin{tabular}{|c|c|c|c|}
\hline$\Gamma_{n \pi}^{\dagger}$ & $\Gamma_{n \nu}^{\dagger}$ & $\Gamma_{g_{9 / 2}}^{\dagger}(\nu)$ & $\Gamma_{f_{5 / 2}}^{\dagger}(\nu)$ \\
\hline 0,02 & 0,50 & 0,17 & 0,33 \\
\hline
\end{tabular}

neutrons :

$$
\begin{aligned}
\Gamma_{n}^{\dagger} & =\Gamma_{n \pi}^{\dagger}+\Gamma_{n \nu}^{\dagger}, \\
\Gamma_{p}^{\dagger}(k) & =\left\langle\tilde{R}_{n p b} \mid R_{n p b}\right\rangle_{k} \Gamma_{p b}(k), \\
\Gamma_{n k}^{\dagger} & =\sum_{p} \Gamma_{p}^{\uparrow}(k),
\end{aligned}
$$

onde $k$ é um índice que rotula o tipo de partícula $(k=\pi$ ou $k=\nu)$. Na tab.1.6 apresentamos as larguras parciais para $E_{n}=15,84 \mathrm{MeV}$ e $\Gamma_{n}^{\uparrow}=0,52 \mathrm{MeV}$.

As análises [Bo 89] dos espectros de prótons e neutrons em termos 
do cálculo estatístico de Hauser-Feshbach, mostram que o decaimento da RGE0 do $Z r^{90}$ por emissão de prótons é compatível com o decaimento estatístico, e que o canal de neutrons apresenta $\sim 5 \%$ de decaimento semi-direto populando o buraco $g_{9 / 2}^{-1}$ do $Z r^{90}$. Estes resultados são compatíveis com os nossos (tab1.6), exceto pela população do buraco $f_{5 / 2}^{-1}$, onde os nossos resultados apresentam uma largura de $0,33 \mathrm{MeV}$. No próximo capítulo apresentaremos uma análise do decaimento deste modo de excitação em termos do modelo híbrido [Di 86b], dentro da aproximação de desacoplamento dos canais de partículas emitidas (os possíveis canais de saída são os de prótons, neutrons e alfas). Nesta análise, a largura de escape obtida $(\sim 0,44 \mathrm{MeV})$ apresenta um bom acordo com a largura obtida na presente seção $(0,52 \mathrm{MeV}$, conforme tab.1.6).

Os resultados apresentados neste capítulo, mostram que a inclusão do contínuo através das energias complexas das ressonâncias de partícula-única [Pi 87, Te 91a], fornecem resultados bastantes satisfatórios em relação aos dados experimentais, mostrando que os efeitos de escape das RG's podem ser bem descritos com a utilização das larguras dos estados de partícula que estão no contínuo. 


\section{Capítulo 2}

\section{Decaimento das ressonâncias}

\section{gigantes}

As observações experimentais dos espectros de partículas emitidas no decaimento das ressonâncias gigantes (RG), fornecem informações sobre as larguras de escape $\left(\Gamma^{\dagger}\right)$ e as larguras de distribuição $\left(\Gamma^{\downarrow}\right)$ destes modos de excitações nucleares, sendo a largura total $\Gamma=\Gamma^{\dagger}+\Gamma^{\downarrow}$. A largura de escape $\left(\Gamma^{\dagger}\right)$, decaimento semi-direto, nos fornece informações sobre o acoplamento das excitações do tipo 1partícula-1buraco (1p-1b) ao contínuo, deixando o núcleo residual em um estado de buraco. Por outro lado, a largura de distribuição $\left(\Gamma^{\downarrow}\right)$, é o resultado da evolução das excitações $1 \mathrm{p}-1 \mathrm{~b}$ para excitações mais complexas $(2 \mathrm{p}-2 \mathrm{~b}, \ldots)$, até que o sistema alcance o equilíbrio no núcleo composto (NC), onde a memória do processo de formação é perdida (exceto pela conservação dos números quânticos). O decaimento do NC é estudado através dos cálculos estatísticos de Hauser-Feshbach 
(HF) [Ha 52], no qual a partícula emitida pode popular qualquer nível acessível, energeticamente, do núcleo residual. No apêndice $C$ apresentamos um breve resumo sobre este tipo de cálculo. Em cada estágio de evolução das excitações de partículaburaco, pode haver acoplamento ao contínuo, e este modo de decaimento é chamado de pré-equilíbrio.

$$
\text { O modelo híbrido [Di 86b] para o decaimento (apêndice C), leva em }
$$
conta tanto o decaimento semi-direto quanto o estatístico, simultâneamente, onde a componente estatística é normalizada a um parâmetro de mistura $\mu$ que mede o grau de fragmentação da $R G\left(\mu=\Gamma^{\downarrow} / \Gamma\right)$. Porém, quando temos mais do que um tipo de partícula possível de ser emitida pela RG, p.ex. prótons e nêutrons, fica difícil de se controlar e de se entender o parâmetro de mistura $\mu$, ou seja, se em um dos canais o decaimento for estatístico, e no outro houver uma contribuição semi-direta, torna-se complicada a avaliação do valor de $\mu$. A estimativa das contribuições semi-diretas, pode ser obtida através da comparação do cálculo estatístico com o experimento, quando o decaimento estatístico é dominante, onde uma subestimação na população dos estados de buraco do núcleo residual, pelo cálculo estatístico,é suprida pela contribuição semi-direta. Uma possível sepàração dos canais [Te 93c] dentro do formalismo do modelo híbrido, implicaria numa maior transparência nas análises dos espectros dos canais envolvidos no decaimento. Assim, para cada tipo de partícula teríamos um parâmetro $\mu$ correspondente. Dentro deste contexto, vamos fazer a análise do decaimento da ressonância gigante de monopolo (RGE0) do $Z r^{90}$, onde há competição entre os canais de prótons e neutrons. 


\subsection{O modelo híbrido para competição entre}

\section{canais}

Dentro do formalismo do modelo híbrido [Di 86b] (vide apêndice C), a população de um certo nível do núcleo residual pela partícula ejetada do núcleo excitado na RG, é dada por:

$$
b_{i}=(1-\mu) \frac{\tau_{i D}}{\sum_{j} \tau_{j D}}+\mu \frac{\tau_{i c}+\mu \tau_{i D}}{\sum_{j}\left(\tau_{j c}+\mu \tau_{j D}\right)}
$$

onde $b_{i}$ é a razão de ramificação para a população do i-ésimo nível do núcleo residual, $\tau_{i D}$ é o coeficiente de transmissão devido ao escape para o contínuo através da RG, $\tau_{i c}$ é o coeficiente de transmissão devido ao núcleo composto e $\mu=\Gamma^{\downarrow} / \Gamma\left(\Gamma=\Gamma^{\uparrow}+\right.$ $\Gamma^{\downarrow}$ ) é um parâmetro que mede o grau de fragmentação da RG. Se tivermos mais do que um tipo de partícula sendo emitida pelo núcleo excitado, p.ex. prótons, alfa, neutrons..., temos :

$$
b_{i}^{k}=(1-\mu) \frac{\tau_{i D}^{k}}{\sum_{l} \sum_{j} \tau_{j D}^{l}}+\mu \frac{\tau_{i c}^{k}+\mu \tau_{i D}^{k}}{\sum_{i} \sum_{j}\left(\tau_{j c}^{l}+\mu \tau_{j D}^{l}\right)}
$$

onde $k$ é um índice que rotula o tipo de partícula emitida ( $k=\pi$ para prótons, $k=\nu$ para neutrons,...), e a soma em $l$ significa que os denominadores de 2.2 são somados em todos os canais de partícula permitidos. Da eq.2.2, temos que:

$$
\sum_{k}\left(\sum_{i} b_{i}^{k}\right)=\sum_{k} P_{k}=1
$$

onde $P_{k}=\sum_{i} b_{i}^{k}$ é a probabilidade de que a partícula emitida seja a partícula $k$ (o que pode ser obtido experimentalmente). 
Uma análise dos dados experimentais através da eq.2.2 é um tanto difícil de ser feita, uma vez que devemos controlar o parâmetro $\mu$ para todos os canais envolvidos no decaimento. Desta forma, se conseguíssemos expressar os $b_{i}^{k}$ 's da eq.2.2 por uma expressão equivalente, onde tivéssemos a independência entre os canais de partícula, teríamos uma forma bem mais simples e transparente de se fazer a análise do decaimento para os possíveis canais.

Vamos reescrever a eq.2.2 da seguinte forma:

$$
b_{i}^{k}=(1-\mu) P_{D}^{k} \frac{\tau_{i D}^{k}}{\sum_{j} \tau_{j D}^{k}}+\mu P_{c}^{k} \frac{\tau_{i c}^{k}+\mu \tau_{i D}^{k}}{\sum_{j}\left(\tau_{j c}^{k}+\mu \tau_{j D}^{k}\right)}
$$

onde :

$$
P_{D}^{k}=\frac{\sum_{j} \tau_{j D}^{k}}{\sum_{l}\left(\sum_{j} \tau_{j D}^{l}\right)} \Rightarrow \sum_{k} P_{D}^{k}=1
$$

e

$$
P_{c}^{k}=\frac{\sum_{j}\left(\tau_{j c}^{k}+\mu \tau_{j D}^{k}\right)}{\sum_{l}\left[\sum_{j}\left(\tau_{j c}^{l}+\mu \tau_{j D}^{k}\right)\right]} \Rightarrow \sum_{k} P_{c}^{k}=1
$$

$\left(P_{D}^{k}\right.$ e $P_{c}^{k}$ são as ramificações, para um canal $k$ específico, devido as contribuições semi-diretas e estatísticas, respectivamente).

A probabilidade $P_{k}$ pode ser obtida somando os $b_{i}^{k}$ s da eq. $2.3 \mathrm{em}$ todos os níveis do núcleo residual que podem ser populados pela partícula $k$ :

$$
P_{k}=\sum_{i} b_{i}^{k}=(1-\mu) P_{D}^{k}+\mu P_{c}^{k}
$$

$\operatorname{com} \sum_{k} P_{k}=1$

$$
\text { Assumindo que } \Gamma=\Gamma^{\uparrow}+\Gamma^{\downarrow} \text {, temos que } \mu=\Gamma^{\downarrow} / \Gamma \text { pode ser }
$$

reescrito como:

$$
\mu=1-\frac{\Gamma^{\dagger}}{\Gamma}
$$


Sendo que a largura de escape, $\Gamma^{\uparrow}$, pode ser escrita como uma soma das larguras parciais das partículas $k$ 's (conforme eq.1.49) :

$$
\Gamma^{\uparrow} \approx \sum_{k} \Gamma_{k}^{\uparrow}
$$

e como para as partículas $p$ no contínuo, emitidas via $R G$, temos que $\tau_{p D}=2 \pi \rho_{D} \Gamma_{p}^{\dagger}$ [Di 86b, Ta 90$]$ ( $\rho_{D}$ é a densidade de estados de $1 \mathrm{p}-1 \mathrm{~b}$ ), podemos escrever a eq.2.5 como:

$$
P_{D}^{k}=\frac{\Gamma_{k}^{\dagger}}{\Gamma^{\dagger}}
$$

Da eq.2.10, pode-se observar que: de todas as partículas que escapam para o contínuo via $\mathrm{RG}\left(\Gamma^{\uparrow}\right), P_{D}^{k}$ é a probabilidade de escape para a partícula $k$. Agora, vamos definir algumas grandezas que possibilitarão a separação dos canais de partículas na eq. 2.4. Das eqs. 2.8 e 2.10 , temos:

$$
(1-\mu) P_{D}^{k}=\frac{\Gamma_{k}^{\dagger}}{\Gamma}=\left(1-\mu_{k}\right) P_{k}
$$

cuja substituição na eq.2.7, fornece:

$$
\mu P_{c}^{k}=\mu_{k} P_{k}
$$

onde definimos:

$$
\mu_{k}=1-\frac{1}{P_{k}} \frac{\Gamma_{k}^{\dagger}}{\Gamma}
$$

como sendo um parâmetro (análogo ao $\mu$ da eq.2.2) que mede a componente estatística do decaimento pela partícula $k$. Então, a eq.2.4 pode ser reescrita como:

$$
b_{i}^{k}=\left(1-\mu_{k}\right) P_{k} \frac{\tau_{i D}^{k}}{\sum_{j} \tau_{j D}^{k}}+\mu_{k} P_{k} \frac{\tau_{i c}^{k}+\mu \tau_{i D}^{k}}{\sum_{j}\left(\tau_{j c}^{k}+\mu \tau_{j D}^{k}\right)} .
$$


$\mathrm{Na}$ eq.2.14, temos uma forma bem mais simplificada para a análise do decaimento (via partícula $k$ ) do que na eq.2.2, uma vez que $P_{k}$ pode ser obtida dos dados experimentais. Porém na eq.2.14 ainda temos os efeitos dos vários canais $k$ presentes no parâmetro $\mu$ do segundo termo do lado direito da eq.2.14. Vamos chamar este termo de $S_{i c}^{k}$ :

$$
S_{i c}^{k}=\mu_{k} P_{k} \frac{\tau_{i c}^{k}+\mu \tau_{i D}^{k}}{\sum_{j}\left(\tau_{j c}^{k}+\mu \tau_{j D}^{k}\right)} .
$$

Utilizando $\sum_{k} P_{c}^{k}=1$, como definido em 2.6, podemos obter o parâmetro $\mu$, fazendo a soma em $k$ na eq.2.12, como sendo composto pelas várias componentes que participam no decaimento, fornecendo :

$$
\mu=\sum_{k} \mu_{k} P_{k} .
$$

Desta forma, reescrevendo a eq.2.15, e separando os canais $l \neq k$ para $\mu$, na expressão acima, obtemos:

$$
S_{i c}^{k}=\mu_{k} P_{k}\left\{\frac{\left(\tau_{i c}^{k}+\mu_{k} P_{k} \tau_{i D}^{k}\right)+\sum_{l \neq k} \mu_{l} P_{l} \tau_{i D}^{k}}{\sum_{j}\left[\left(\tau_{j c}^{k}+\mu_{k} P_{k} \tau_{j D}^{k}\right)+\sum_{l \neq k} \mu_{l} P_{l} \tau_{j D}^{k}\right]}\right\} .
$$

$\mathrm{Na}$ eq.2.17, vê-se claramente que a interferência entre canais, presentes em $\mu$, está contida no termo $\sum_{l \neq k} \mu_{l} P_{l} \tau_{i D}^{k}$ de $S_{i c}^{k}$. Se tivermos uma situação onde o decaimento estatístico domine, e que haja dominância de um dos canais de partícula, p.ex. em [Bo 89] $\left(\operatorname{EO}\left(Z r^{90}\right)\right)$, o canal de neutrons é da ordem de $88 \%$ e é dominantemente estatístico, podemos desprezar os efeitos de interferência e escrever:

$$
S_{i c}^{k} \approx \mu_{k} P_{k} \frac{\tau_{i c}^{k}+\mu_{k} P_{k} \tau_{i D}^{k}}{\sum_{j}\left(\tau_{j c}^{k}+\mu_{k} P_{k} \tau_{j D}^{k}\right)} .
$$


Agora, podemos obter uma expressão aproximada para os $b_{i}^{k}$ 's da eq.2.2, onde os canais de partícula são tratados independentemente uns dos outros:

$$
b_{i}^{k} \approx\left(1-\mu_{k}\right) P_{k} \frac{\tau_{i D}^{k}}{\sum_{j} \tau_{j D}^{k}}+\mu_{k} P_{k} \frac{\tau_{i c}^{k}+\mu_{k} P_{k} \tau_{i D}^{k}}{\sum_{j}\left(\tau_{j c}^{k}+\overline{\left.\mu_{k} P_{k} \tau_{j D}^{k}\right)}\right.}
$$

Uma análise com a aplicação da eq.2.19, é bem mais simples e transparente do que aquela da eq.2.2, pois o espectro de cada partícula vai nos fornecer seu correspondente $\mu_{k}$, o qual vai medir o quanto do referido decaimento é estatístico, estando este tipo de informação um tanto obscuro na eq.2.2. Para cada espectro temos um $\mu_{k}$, e a partir deles podemos obter da eq.2.13, as larguras de escape $\Gamma_{k}^{\uparrow}$ correspondentes, e em consequência $\Gamma^{\dagger}$. Tendo todos os $\mu_{k}$ 's possiveis, obtemos da eq.2.16 o parâmetro $\mu$ da eq.2.2.

A unitariedade do modelo híbrido [Di 86b] é preservada na separação dos canais da eq.2.14, mas não é obtida ao desprezarmos as interferências entre os canais na eq.2.19. Entretanto, nesta equação, também temos uma unitariedade. Esta é obtida, porque estamos "forçando" a componente estatística ( $S_{i c}^{k}$ na eq.2.18) a conter apenas contribuiçôes próprias ao seu específico canal. Tal unitariedade "forçada" vem no mesmo sentido das aproximações feitas nas análises em

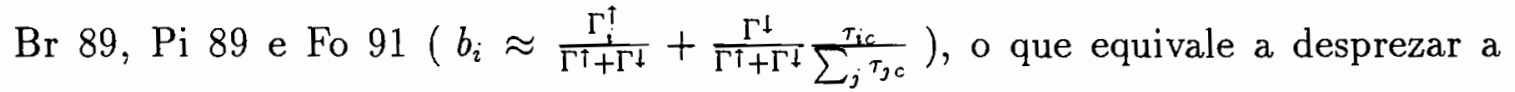
contribuição semi-direta no segundo termo do lado direito da eq.2.1 $\left(\mu \sum_{j} \tau_{j D}\right)$, em comparação com a contribuição estatística de Hauser-Feshbach $\left(\sum_{j} \tau_{j c}\right)$. Porém, uma análise com estas aproximações, apresenta menos problemas do que aquelas da eq.2.1. 
Os problemas decorrentes do uso da eq.2.1, provém da determinação das larguras parciais de escape $\Gamma_{i}^{\uparrow}$, calculadas através dos coeficientes de transmissão $\tau_{i D}\left(\tau_{i D}=2 \pi \rho_{D} \Gamma_{i}^{\uparrow}\right)$. Dependendo do valor de $\rho_{D}$ utilizado, obtém-se uma certa largura de escape $\Gamma^{\dagger}$ [Br 88]. Nos trabalhos de Dias e outros [Di 86b] e Bracco e outros $[\mathrm{Br} 88]$, utiliza-se $\rho_{D}=1 \mathrm{MeV}^{-1}$ para obterem a largura de escape, mas Piza e outros [ $\mathrm{Pi} 89]$ determinam $\rho_{D}=1 / \pi^{2} \Gamma$, que no caso das RG's, as quais tem larguras da ordem de $\mathrm{MeV}$, fornece $\rho_{D} \sim 10^{-1} \mathrm{MeV}^{-1}$.

Desta forma, uma análise a partir do cálculo estatístico de HauserFeshbach, resultaria em fazermos a aproximação $\frac{\tau_{i c}^{k}+\mu \tau_{j D}^{k}}{\sum_{j}\left(\tau_{j c}^{k}+\mu \tau_{j D}^{k}\right)} \approx \frac{\tau_{i c}^{k}}{\sum_{j}^{k} \tau_{j c}^{k}}$ na eq.2.14. Assim, já estaríamos desprezando os efeitos de interferência entre os canais na eq.2.17, o que consiste em tomarmos $\frac{\sum_{j} \tau_{j D}^{k}}{\sum_{j} \tau_{j c}^{k}} \ll 1$. A aproximação é boa quando o decaimento estatístico domina.

As incertezas ocasionadas por $\rho_{D}$, em $\tau_{i D}^{k}$, no primeiro termo das eqs.2.1 ou 2.19 , não vão acarretar problema algum, porque nele há uma razão entre os coeficientes de transmissão semi-diretos $\left(\frac{\tau_{i D}^{k}}{\sum_{j}^{i} \tau_{j D}^{k}}=\frac{\Gamma_{i k}^{\dagger}}{\Gamma_{k}^{\dagger}}\right)$, não havendo a dependência com $\rho_{D}$.

\subsection{Larguras de escape da RGE0 no $Z r^{90}$}

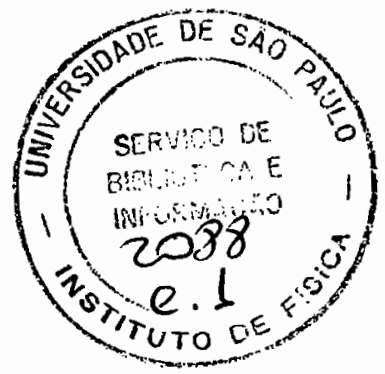

Nesta seção vamos analisar os dados experimentais [Bo 89] da reação $Z r^{90}\left(\alpha, \alpha^{\prime}, k\right)$, onde o espectro de cada partícula $k$ emitida, é medido em coincidência com a partícula $\alpha^{\prime}$ espalhada. Os possíveis canais de saída ( $k$ ) são 
Tabela 2.1: Possíveis canais de saída na RGE0 do $Z r^{90}$, as probabilidades $P_{k}$ 's são valores extraídos dos resultados experimentais.

\begin{tabular}{|c|c|c|}
\hline $\mathrm{k}$ & $\operatorname{limiar}(\mathrm{MeV})$ & $P_{k}(\%)$ \\
\hline nêutron $(\nu)$ & 11,98 & 88 \\
\hline $\operatorname{próton~}(\pi)$ & 8,36 & 10 \\
\hline $\operatorname{alfa}(\alpha)$ & 6,68 & 2 \\
\hline
\end{tabular}

dados na tab.2.1, juntamente com as probabilidades $P_{k}$ 's e seus limiares de emissão. Os resultados experimentais fornecem os espectros para prótons e neutrons, e serão estes os canais que iremos estudar.

O estudo do decaimento do $Z r^{90}$ é interessante na medida em que neste núcleo, o decaimento das RG's envolve mais do que um tipo de partícula emitida, havendo competição entre estes canais, o que para núcleos pesados como o $P b^{208}$, não é possível devido a forte repulsão coulombiana para partículas carregadas. Por outro lado, o emprego da eq.2.19 nestes cálculos, necessita dos conhecimentos das energias, spins e paridades dos níveis dos núcleos residuais, o que para o $Z r^{90}$, podem ser conseguidos por algum modelo simples de estrutura nuclear. O $Z r^{90}$ apresenta camada fechada para neutrons e subcamada fechada para prótons, permitindo a descrição dos núcleos residuais ( $Y^{89}$ para $k=\pi$ e $Z r^{89}$ para $k=\nu$ ) pelo modelo partícula-vibrador, com o caroço sendo o $S^{88}$ (vide apêndice B). Um outro 
ponto de interesse é que podemos estudar a aplicabilidade e a transparência do modelo híbrido quando fazemos a separação entre canais, conforme o tratamento dado na seção anterior, o que nos permite fazer uma estimativa das larguras de escape $\left(\Gamma^{\dagger} \approx \Gamma_{\pi}^{\dagger}+\Gamma_{\nu}^{\dagger}\right)$ para compararmos com os resultados obtidos pela RPA no contínuo da sec.1.2.

As energias e larguras experimentais [Bo 89] da RGE0 no $Z r^{90}$, são $\quad \bar{E}_{x}=(16,1 \pm 0,4) \mathrm{MeV}$ e $\Gamma=(3,1 \pm 0,4) \mathrm{MeV}$, esgotando $48 \%$ da EWSR. Os espectros de prótons e neutrons foram medidos no intervalo de energia $14,75<E_{x}\left(Z r^{90}\right)<17,75 \mathrm{MeV}$. No trabalho experimental [Bo 89], das análises destes espectros feitas com o cálculo estatístico, os autores concluiram que o espectro de prótons é compatível com os cálculos, e que o de neutrons apresenta cerca de $5 \%$ de decaimento semi-direto, que popula o estado fundamental $\left(j^{\pi}=9 / 2^{+}\right)$do $Z r^{89}$. Nestes cálculos, eles [Bo 89] utilizaram níveis discretos em baixas energias dos núcleos residuais $\left(0 \leq E_{x}\left(Y^{89}\right) \leq 3,5 \mathrm{MeV}\right.$ e $\left.0 \leq E_{x}\left(Z r^{89}\right) \leq 2,1 \mathrm{MeV}\right)$, e acima delas, empregaram uma densidade de níveis tipo gás de Fermi [Di 73], com parâmetros ajustados a estes núcleos. Os parâmetros do potencial ótico que utilizaram, foram os de Rapaport e outros [Ra 79].

Os espectros experimentais, e seus respectivos cálculos estatísticos [Bo 89], são apresentados nas figs. 2.1 e 2.2, para prótons e neutrons, respectivamente. Nestas figuras os espectros calculados (linha contínua) foram ajustados com a eficiência dos detetores $(200 \mathrm{KeV})$ e a resolução em que as partículas foram medidas $(500 \mathrm{KeV})$. O espectro experimental das partículas alfas, $k=\alpha$, não foi 


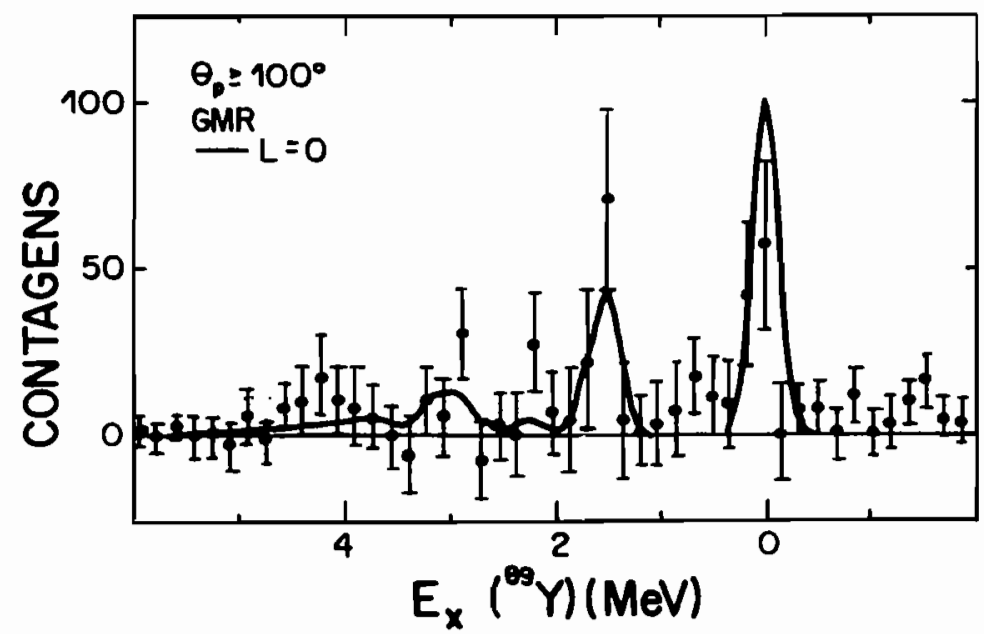

Figura 2.1: Espectro experimental de prótons para a RGE0 do $Z r^{90}$.

construído devido ao baixo número de partículas detetadas $\left(P_{\alpha}=2 \%\right)$.

A seguir apresentaremos nossos resultados, onde os espectros experimentais são analisados de acordo com o método descrito na seção anterior. Os níveis de energia dos núcleos residuais necessários ao cálculo da componente estatística, são apresentados no apêndice B. Os cálculos são feitos no centróide de energia, $E_{x}=16,25 \mathrm{MeV}$, do intervalo em que as partículas foram detetadas. Os coeficientes de transmissão, $\tau_{i c}^{k}$, são obtidos através do código computacional SCAT [Be 79], utilizando o potencial ótico de Rapaport e outros[Ra 79]. Desprezamos as contribuições semi-diretas $\left(\tau_{i D}^{k}\right)$ em $S_{i c}^{k}$ da expressão 2.18 , uma vez que o denominador de Hauser-Feshbach $\left(\sum_{j} \tau_{j c}^{k}\right)$ deve ser muito grande comparado a $\mu_{k} P_{k} \sum_{j} \tau_{j D}^{k}$ quando o decaimento estatístico é dominante. Resumindo, estamos desprezando a 


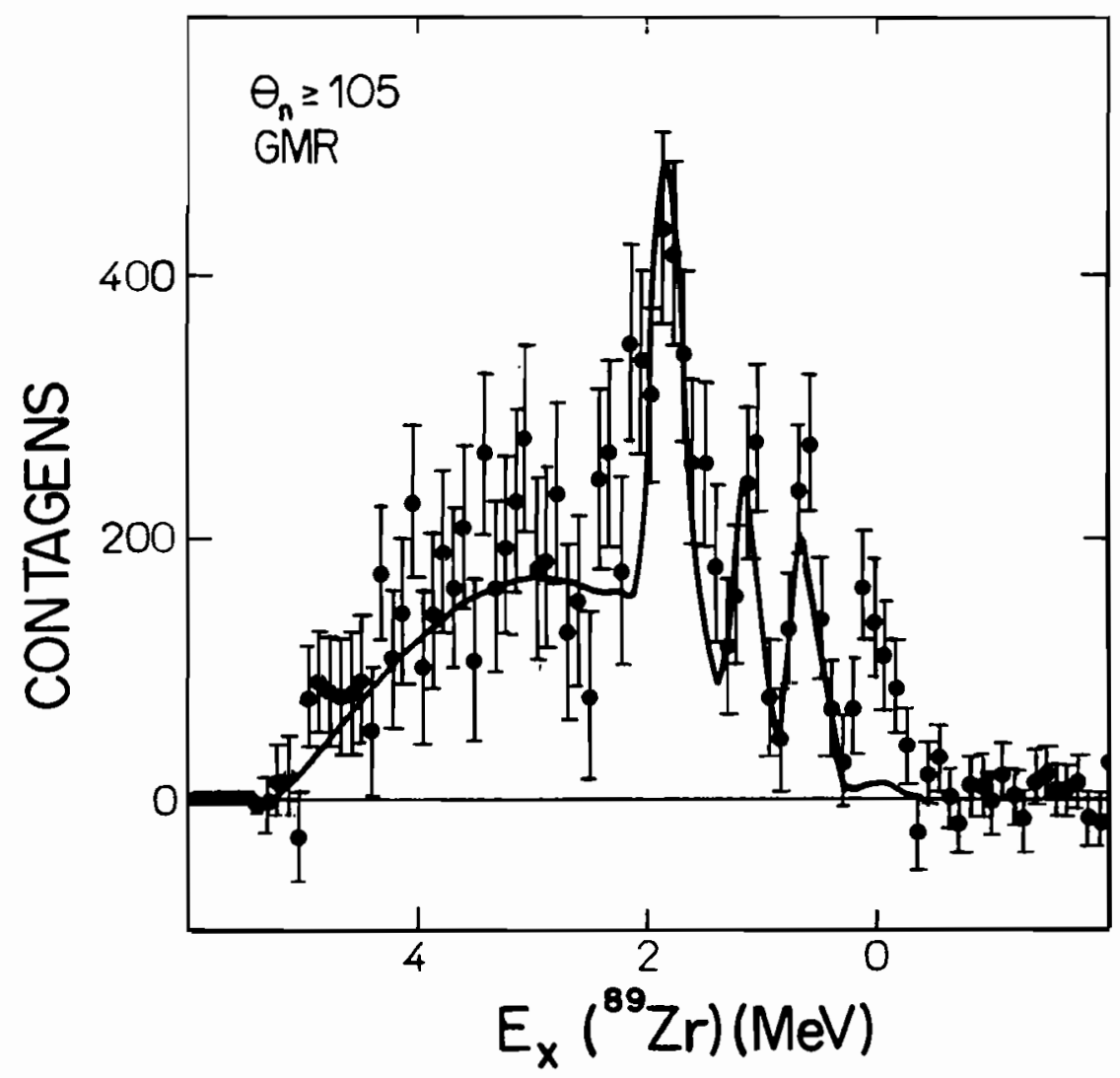

Figura 2.2: Espectro experimental de neutrons para a RGE0 do $Z r^{90}$.

componente semi-direta no segundo termo do lado direito da eq.2.19.

Sendo assim, em nossas análises, a população dos estados de buraco do núcleo residual, provenientes do decaimento semi-direto, é governado pelo primeiro termo do lado direito da expressão 2.19 , ou seja:

$$
b_{i}^{k} \approx\left(1-\mu_{k}\right) P_{k} \frac{\tau_{i D}^{k}}{\sum_{j} \tau_{j D}^{k}}+\mu_{k} P_{k} \frac{\tau_{i c}^{k}}{\sum_{j} \tau_{j c}^{k}},
$$

com $P_{k}$ dado na tab.2.1. 


\subsection{Canal de prótons $(k=\pi)$}

Neste canal, referente ao decaimento da RGE0 do $Z r^{90}$ por emissão de prótons, o núcleo residual é o $Y^{89}$, o qual pode ser populado até $E_{x}\left(Y^{89}\right)=$ $7,89 \mathrm{MeV}$. Devido à forte repulsão coulombiana, os coeficientes de transmissão começam a contribuir [Bo 89] a partir da energia de próton $\varepsilon_{\pi} \approx 4,0 \mathrm{MeV}$, o que pode ser observado nas figs. 2.1 e 2.3, para o espectro experimental e os coeficientes de transmissão, respectivamente. Observa-se ainda que os níveis de energia do $Y^{89}$ abaixo de $2,0 \mathrm{MeV}$ são os mais fortemente populados, sendo o decaimento dominante para os prótons de mais altas energias, ao contrário do que ocorre para o canal de neutrons (vide fig.2.2). Por causa deste efeito, os níveis de energia do $Y^{89}$ relevantes no cálculo, são aqueles com energias na região de $E_{x}\left(Y^{89}\right)<4,5 \mathrm{MeV}$, pois como vemos na fig.2.1, a população do $Y^{89}$ se dá até $\sim 4,5 \mathrm{MeV}$.

Fazendo $\mu_{\pi}=1$ e $P_{\pi}=0,1$ na expressão 2.20 , e utilizando os níveis experimentais [NDS] do $Y^{89}$ até $E_{x}\left(Y^{89}\right)=3,0 \mathrm{MeV}$, juntamente com os níveis previstos teóricamente (apêndice B) no intervalo de energia $3,0<E_{x}\left(Y^{89}\right)<6,6 \mathrm{MeV}$, obtivemos o espectro de prótons do decaimento da $\operatorname{RGE0}\left(Z r^{90}\right)$ apresentado na fig.2.4. Nesta figura, cada linha de prótons é representada por uma gaussiana de largura a meia altura $\mathrm{FWHM}=400 \mathrm{KeV}$, proveniente do ajuste do espectro calculado com o resultado experimental. A normalização do cálculo estatístico é feita na região de energia dos prótons que populam o $Y^{89}$ entre $2,0 \leq E_{x}\left(Y^{89}\right) \leq 4,5 \mathrm{MeV}$, a qual está fora do intervalo onde uma possível contribuição semi-direta possa ocor- 


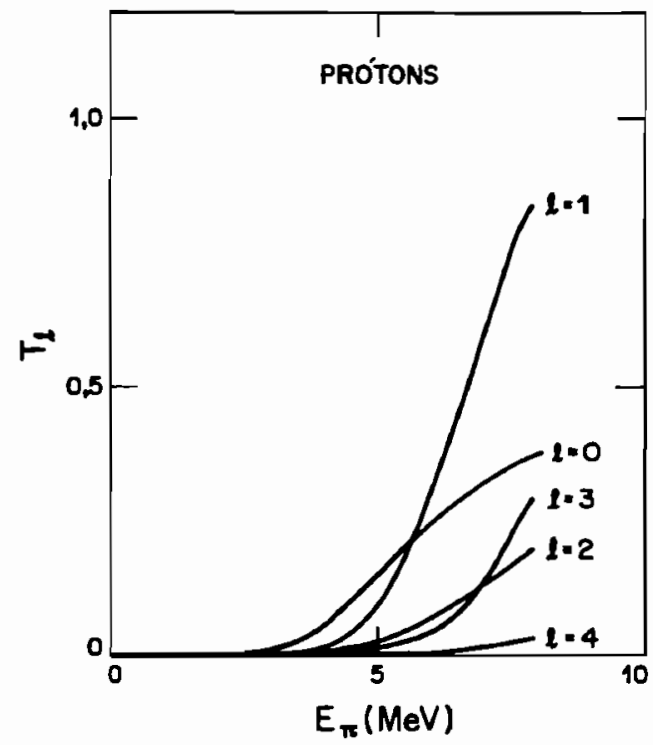

Figura 2.3: Coeficientes de transmissão para os prótons provenientes do decaimento da RGE0 do $Z r^{90}$.

rer (os buracos do $Y^{89}$ estão em $E_{x}<2.0 \mathrm{MeV}$, conforme apêndice B). Com esta normalização obtivemos que o número total de prótons previstos pelo cálculo, entre $0 \leq E_{x}\left(Y^{89}\right) \leq 4.5 \mathrm{MeV}$, apresenta um bom acordo com o espectro experimental, conforme mostra a fig.2.4.

Os resultados obtidos, mostram que o decaimento da $\operatorname{RGE0}\left(Z r^{90}\right)$ por emissão de prótons é razoavelmente bem descrito por um cálculo estatístico de Hauser-Feshbach $\left(\mu_{\pi}=1\right)$, concordando com o resultado obtido no trabalho experimental [Bo 89]. Na comparação do nosso cálculo estatístico com os dados experimentais, mostrados na fig.2.4, devem ser salientados três pontos:

(1) no espectro experimental aparentemente existe uma preferência da população 


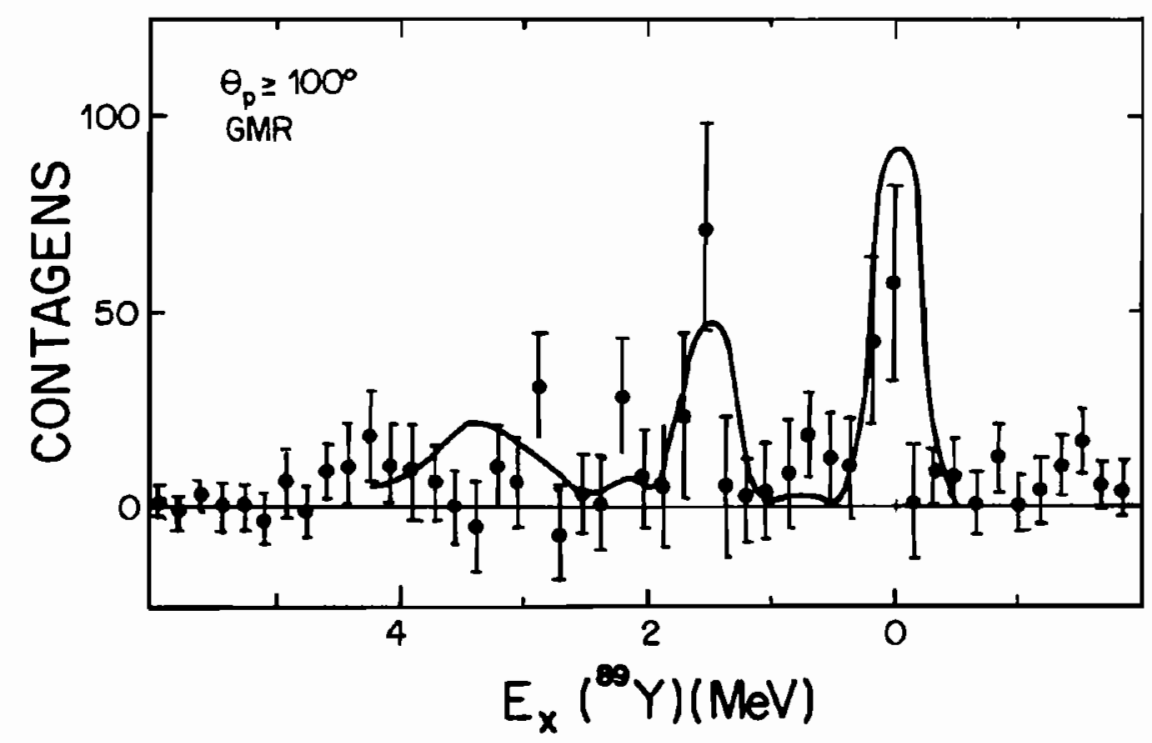

Figura 2.4: Espectro de prótons teórico (linha contínua) em comparação com o experimental (circulos cheios).

do pico em $E_{x}\left(Y^{89}\right) \approx 1,5 \mathrm{MeV}$ (um buraco com $j^{\pi}=3 / 2^{-}$) em relação ao estado fundamental ( $\left.j^{\pi}=1 / 2^{-}\right)$, enquanto que no cálculo estatístico isto não ocorre (vide figs2.1 e 2.4). Estes dois níveis são populados por prótons de mesmo momento angular $(l=1)$ a energias diferentes, sendo a contribuição para o estado fundamental maior do que para o estado $j^{\pi}=3 / 2^{-}$, uma vez que $\varepsilon_{\pi}\left(3 / 2^{-}\right)=6,4 \mathrm{MeV}<$ $\varepsilon_{\pi}\left(1 / 2^{-}\right)=7,89 \mathrm{MeV}$, vide fig.2.3 para os $T_{l}$ 's dos prótons;

(2) a população do estado $9 / 2^{+}$em $E_{x}\left(Y^{89}\right) \approx 0,91 \mathrm{MeV}$ é fracamente reproduzida pelos nossos cálculos, o que pode ser notado na fig.2.4. Este desacordo também ocorre no cálculo estatístico do trabalho experimental, como pode ser observado na fig. 2.1 ; 
(3) finalmente, salientamos que o deslocamento do pico teórico, previsto a $3,5 \mathrm{MeV}$, em relação aos picos experimentais em $E_{x}\left(Y^{89}\right)=3,0 \mathrm{e} 4,0 \mathrm{MeV}$, pode ser explicado por um possível deslocamento das energias previstas em nosso modelo teórico para os níveis do $Y^{89}$, onde existe uma concentração de níveis com spins baixos $\left(j^{\pi} \leq 5 / 2\right)$ por volta de $E_{x}\left(Y^{89}\right) \approx 3,5 \mathrm{MeV}$ (vide tab.B.4 do apêndice B). Daí o pico, em nosso cálculo, nesta energia de excitação do $Y^{89}$.

Apesar dos pontos levantados acima, na comparação dos nossos cálculos com os dados experimentais, podemos concluir que o decaimento da RGE0 $\left(Z r^{90}\right)$ por emissão de prótons, pode ser explicado razoavelmente bem, assumindo $\mu_{\pi}=1$ ( decaimento estatístico) na expressão 2.20. Com este resultado obtemos a largura de escape para o canal de prótons através da eq.2.13 (utilizando a largura experimental $\Gamma=3,1 \mathrm{MeV}$ ), a qual fornece que para $\mu_{\pi} \approx 1$ temos $\Gamma_{\pi}^{\dagger} \approx 0 \mathrm{MeV} . \mathrm{A}$ análise do espectro de prótons através do decaimento estatístico, mostra um razoável acordo com o obtido no cálculo das larguras de escape com a RPA no contínuo, onde obtivemos $\Gamma_{\pi}^{\dagger}(R P A) \approx 0,020 \mathrm{MeV}$ correspondendo a $\mu_{\pi}(\mathrm{RPA}) \approx 0,935$ (pela eq.2.13).

\subsection{Canal de neutrons $(k=\nu)$}

Os neutrons emitidos pela RGE0 $\left(Z r^{90}\right)$ em $E_{x}=16,25 \mathrm{MeV}$, irão popular os níveis de energia do núcleo residual $Z r^{89}$ até $E_{x}\left(Z r^{89}\right)=4,27 \mathrm{MeV}$. Utilizamos os níveis de energia experimentais [NDS] até $E_{x}\left(Z r^{89}\right)=2,1 \mathrm{MeV}$, e 
para $E_{x}\left(Z r^{89}\right)>2,1 \mathrm{MeV}$ utilizamos os níveis previstos pelo modelo teórico dado no apêndice B. Dos resultados do cálculo estatístico do trabalho experimental [Bo 89] (vide fig.2.2), podemos observar que existe uma falta de contagem de neutrons rápidos para os estados de buraco do $Z r^{89}$ em $E_{x}\left(9 / 2^{+}\right)=0,0 \mathrm{MeV}, E_{x}\left(1 / 2^{-}\right)=$ $0,59 \mathrm{MeV}, E_{x}\left(3 / 2^{-}\right)=1,09$ e $E_{x}\left(5 / 2^{-}\right)=1,45 \mathrm{MeV}$, dando evidências de que neste canal existe uma contribuição do decaimento semi-direto. Em Bo 89, os autores concluiram que a contribuição semi-direta é da ordem de $5 \%$, e que ela popula somente o estado fundamental $\left(j^{\pi}=9 / 2^{+}\right)$. A falta de contagem dos neutrons que populam o estado fundamental através do cálculo estatístico é claramente visível na fig.2.2. Por outro lado, a subestimação da população dos estados $j^{\pi}=1 / 2^{-}, 3 / 2^{-} \mathrm{e}$ $5 / 2^{-}$não é muito aparente. Analisando a fig. 2.2 na região de energia em torno de $E_{x}\left(Z r^{89}\right) \approx 3,0 \mathrm{MeV}$, observamos que o cálculo estatístico de Bo 89 não reproduz muito bem o espectro experimental. A razão para isso é que a densidade de níveis que eles utilizaram no cálculo, deve subestimar o número de níveis com spins baixos ( $j \leq 5 / 2$ ), acarretando uma subestimação (os autores avaliam esta subcontagem em $\sim 3 \%$ ) na contagem dos neutrons estatísticos nesta região de energia. Nesta região $(\simeq 3,0 \mathrm{MeV})$, o cálculo estatístico deveria reproduzir razoavelmente bem o espectro experimental. Esta subcontagem dos neutrons estatísticos, pode afetar a análise da população dos estados de energias mais baixa do $Z r^{89}\left(j^{\pi}=1 / 2^{-}, 3 / 2^{-} \mathrm{e}\right.$ 5/2- ), dando uma contribuição semi-direta para eles.

Levando em conta os pontos salientados acima, sobre a análise estatística do trabalho experimental [Bo 89] (fig.2.2), fizemos a análise do espectro 
de neutrons, incluindo contribuições semi-diretas para os estados de mais baixa energia do $Z r^{89}\left(j^{\pi}=9 / 2^{+}, 1 / 2^{-}, 3 / 2^{-}\right.$e $\left.5 / 2^{-}\right)$. A análise foi feita com o emprego da expressão 2.20. Utilizamos $P_{\nu}=0,88$ (tab.2.1) e os níveis de energia do $Z r^{89}$ divididos em duas regiões: $E_{x}\left(Z r^{89}\right) \leq 2,1 \mathrm{MeV} \Rightarrow$ níveis experimentais [NDS], e para $E_{x}\left(Z r^{89}\right)>2,1 \mathrm{MeV} \Rightarrow$ níveis teóricos (apêndice B), como foi dito no início desta seção . A normalização com os dados experimentais foi feita em relação ao número total de neutrons entre $0 \leq E_{x}\left(Z r^{89}\right) \leq 4,2 \mathrm{MeV}$, que é a região onde os neutrons são emitidos em nossos cálculos. Cada linha de neutrons foi representada por uma gaussiana com largura $\mathrm{FWHM}=0,25 \mathrm{MeV}$ para os neutrons que populam o $Z r^{89}$ até 2,1 $\mathrm{MeV}$, e acima desta energia uma gaussiana com FWHM $=0,5 \mathrm{MeV}$, provenientes do ajuste com o espectro experimental. A avaliação do valor de $\mu_{\nu}$, e das contribuições semi-diretas, foram obtidas de modo que o espectro calculado reproduzisse os picos experimentais de mais baixas energias do $Z r^{89}\left(j^{\pi}=9 / 2^{+}, 1 / 2^{-}, 3 / 2^{-}\right.$e $\left.5 / 2^{-}\right)$, preservando a contagem do número total de neutrons do intervalo $0 \leq E_{x}\left(Z r^{89}\right) \leq 4,2 \mathrm{MeV}$. Os coeficientes de transmissão e o espectro de neutrons, que obtivemos nestes cálculos, são apresentadas nas figs.2.5 e 2.6, respectivamente, sendo que na fig.2.6 o nosso resultado é comparado com o espectro experimental.

Na fig.2.6, podemos observar um bom acordo entre o nosso cálculo e os dados experimentais. $\mathrm{O}$ parâmetro $\mu_{\nu}$ obtido neste ajuste foi de $\mu_{\nu} \approx 0,84$, indicando que aproximadamente $16 \%$ dos neutrons emitidos, populam os estados de buraco do $Z r^{89}$ via decaimento semi-direto. Com este valor de $\mu_{\nu}$, e com a largura 


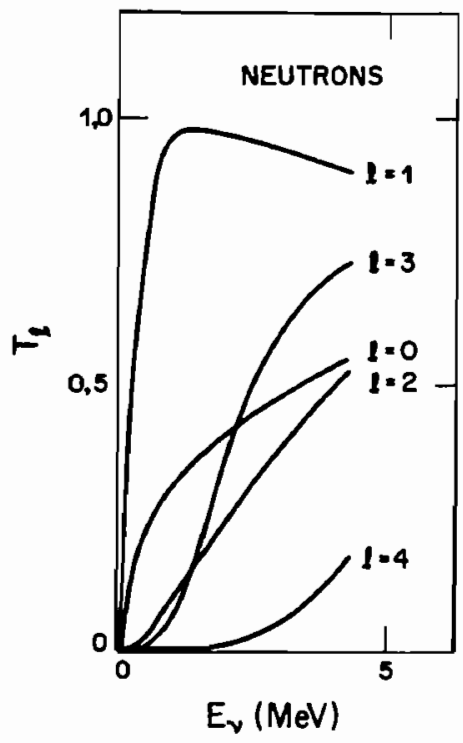

Figura 2.5: Coeficientes de transmissão para os neutrons provenientes do decaimento da RGE0 do $Z r^{90}$.

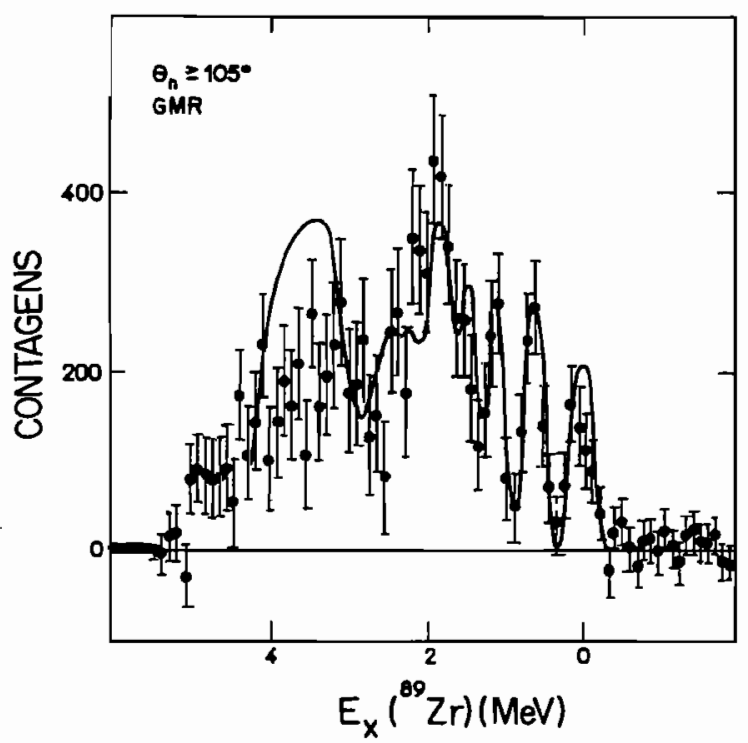

Figura 2.6: Espectro de neutrons teórico (linha contínua) em comparação com o experimental (circulos cheios). 
Tabela 2.2: População dos buracos do $Z r^{89}$ através da contribuição semi-direta.

\begin{tabular}{|c|c|c|c|}
\hline \multicolumn{4}{|c|}{$P_{\nu} \simeq 0,88 ; \mu_{\nu} \simeq 0,84 ; \Gamma \simeq 3,1 \mathrm{MeV} ; \Gamma_{\nu}^{\dagger} \simeq 0,436 \mathrm{MeV}$} \\
\hline $\mathrm{i}$ & $E_{x i}\left(Z r^{89}\right)(\mathrm{MeV})$ & $(\%)$ & $\Gamma_{\nu i}^{\dagger}(\mathrm{MeV})$ \\
\hline $9 / 2^{+}$ & 0,0 & 6 & 0,163 \\
\hline $1 / 2^{-}$ & 0,59 & 3 & 0,082 \\
\hline $3 / 2^{-}$ & 1,09 & 3 & 0,082 \\
\hline $5 / 2^{-}$ & 1,45 & 4 & 0,109 \\
\hline
\end{tabular}

$\Gamma \simeq 3,1 \mathrm{MeV}$ experimental, podemos estimar a largura de escape para os neutrons, através da eq.2.13, como sendo $\Gamma_{\nu}^{\dagger} \approx 0,436 \mathrm{MeV}$. Na tab.2.2 apresentamos a participação, no decaimento, de cada estado de buraco do $Z r^{89}$, populados pelos $16 \%$ de neutrons semi-diretos, assim como as larguras de escape correspondentes.

Comparando nossos resultados com aqueles obtidos da análise estatística do trabalho experimental [Bo 89], observamos um bom acordo para as contribuições semi-diretas, somente na população do estado fundamental do $Z r^{89}\left(j^{\pi}=\right.$ $9 / 2^{+}$). A razão para o desacordo entre os nossos cálculos e os de Bo 89 , na população dos estados $j^{\pi}=1 / 2^{-}, 3 / 2^{-}$e $5 / 2^{-}$, se dá por causa da diferença nas densidades de níveis utilizadas (concluímos que há uma componente semi-direta para esses níveis, e em Bo 89 existem apenas as componentes estatísticas).

Ao contrário do que ocorreu nos cálculos estatísticos de Bo 89 , 
nossos cálculos mostram que a população dos neutrons estatísticos na região em torno de $E_{x}\left(Z r^{89}\right) \approx 3,0 \mathrm{MeV}$, apresenta um excesso desses neutrons em relação ao espectro experimental. Este excesso é da ordem de 5\%, e vai afetar principalmente a população dos estados do $Z r^{89}$ na região de energia em torno de $E_{x}\left(Z r^{89}\right) \approx$ $2,0 \mathrm{MeV}$, onde o nosso cálculo subestima a contagem destes neutrons ( a falta é de $\sim 3 \%)$. Este resultado mostra que em nossa descrição dos níveis de energia do $Z r^{89}$, deve haver um excesso de estados com spins baixos $\left(j^{\pi} \leq 5 / 2\right)$ na região de energia $3,0 \leq E_{x}\left(Z r^{89}\right) \leq 4,0 \mathrm{MeV}$ (vide tab.B.3 do apêndice B). O desvio entre o nosso cálculo e os dados experimentais, vai afetar muito pouco os resultados da tab.2.2, pois os $5 \%$ de excesso de neutrons estatísticos em $3,0 \leq E_{x}\left(Z r^{89}\right)$ serão distribuídos entre a população do pico em $E_{x}\left(Z r^{89}\right) \approx 2,0 \mathrm{MeV}(\sim 3 \%)$, e os quatro picos em energias mais baixas $\left(j^{\pi}=9 / 2^{+}, 1 / 2^{-}, 3 / 2^{-}\right.$e $\left.5 / 2^{-}\right)$, os quais terão a contribuição estatatística aumentada de $\sim 2 \%$ (divididos entre eles), acarretando um decréscimo na contribuição semi-direta desta mesma quantidade. A largura $\Gamma_{\nu}^{\uparrow}$ e o parâmetro $\mu_{\nu}$ da tab.2.2 sofreriam esta pequena correção $(\sim 2 \%)$, o que alteraria muito pouco tais resultados. Desta discussão podemos concluir que os nossos resultados apresentam um bom acordo com os dados experimentais.

A largura de escape para neutrons $(0,436 \mathrm{MeV})$, obtida pela presente análise, também mostra um bom acordo com aquela do cálculo através da RPA no contínuo $(0,500 \mathrm{MeV})$ da sec.1.2. No entanto, a concordância entre as larguras parciais dos buracos $1 / 2-, 3 / 2^{-}$e $5 / 2^{-}$do $Z r^{89}$, nestes dois cálculos, não é satisfatória. $\mathrm{Na}$ presente análise, estima-se estas larguras como sendo da ordem 
de $0,082 \mathrm{MeV}$ para os buracos $p_{1 / 2}^{-1}$ e $p_{3 / 2}^{-1}$, e $0,109 \mathrm{MeV}$ para $f_{5 / 2}^{-1}$, enquanto que o cálculo com a RPA no contínuo fornece uma largura quase nula para os buracos $p_{1 / 2}^{-1}$ e $p_{3 / 2}^{-1}$, e $0,330 \mathrm{MeV}$ para $f_{5 / 2}^{-1}$. A largura de escape dos neutrons, obtida na RPA, foi de $\Gamma_{\nu}^{\uparrow}(\mathrm{RPA}) \simeq 0,500 \mathrm{MeV}$, e utilizando os valores experimentais $\Gamma=3,1 \mathrm{MeV} \mathrm{e}$ $P_{\nu}=0,88$, obtemos pela eq. 2.13 um parâmetro $\mu_{\nu}$ da ordem de $0,817\left(\mu_{\nu}(\mathrm{RPA}) \approx\right.$ 0,817), o qual apresenta um desvio de $\sim 3 \%$ em relação ao valor da tab.2.2.

Apesar da concordância da largura de escape total $\Gamma_{\nu}^{\dagger}$, verificada entre os resultados desta seção com aqueles da RPA no contínuo, o mesmo não ocorre entre as larguras parciais, havendo um bom acordo somente para o buraco $g_{9 / 2}^{-1}$. Este tipo de desacordo, também já foi apontado por Bracco [Br 88$]$, na análise da RGE0 do $P b^{208}$.

\subsection{Cálculo do parâmetro de mistura $\mu$}

A partir das análises efetuadas nas seções anteriores, para o decaimento da $\operatorname{RGE0}\left(Z r^{90}\right)$, vamos agora, avaliar o parâmetro de mistura $\mu$ (eq.2.2) do modelo híbrido [Di 86b], o que é feito dentro do formalismo da separação dos canais de partícula da seção 2.1. Uma análise completa deveria levar em conta também o canal de partículas alfa $(\mathrm{k}=\alpha)$. Porém, como o espectro experimental para esse canal não foi possível de ser construído, devido à baixa contagem das partículas $\alpha$ emitidas ( $P_{\alpha} \approx 2 \%$ ), vamos fazer uma estimativa do parâmetro $\mu$ sem a avaliação do $\mu_{\alpha}$. 
Da eq.2.16, temos para $k=\nu, \pi, \alpha$ :

$$
\mu=\mu_{\nu} P_{\nu}+\mu_{\pi} P_{\pi}+\mu_{\alpha} P_{\alpha},
$$

que dentro desta estimativa, fornece :

$$
\mu \approx \mu_{\nu} P_{\nu}+\mu_{\pi} P_{\pi} .
$$

$\mathrm{O}$ valor de $\mu$ na eq.2.22, pode apresentar um desvio da ordem de $2 \%$ em relação ao que seria obtido se tivéssemos o valor de $\mu_{\alpha}$ na eq.2.21. Com a exclusão do canal $k=\alpha$, temos que o nosso cálculo deve ser normalizado à $P_{\nu}+P_{\pi}$, no sentido da eq.2.3, e não a 1 , como seria o caso se tivéssemos todos os $\mu_{k}$ 's. Sendo assim, as eqs.2.9 e 2.13 fornecerão a largura de escape $\left(\Gamma^{\dagger}\right)$ como uma soma das larguras de escape dos canais $k=\nu$ e $k=\pi$ :

$$
\Gamma^{\uparrow} \approx\left(P_{\pi}+P_{\nu}-\mu\right) \Gamma=\Gamma_{\pi}^{\uparrow}+\Gamma_{\nu}^{\uparrow} .
$$

Substituindo os valores $\mu_{\nu}=0,84, P_{\nu}=0,88, \mu_{\pi}=1$ e $P_{\pi}=0,1$, obtidos da análise do decaimento da $\operatorname{RGE0}\left(Z r^{90}\right)$, obtemos $\mu \approx 0,839$ e $\Gamma^{\uparrow} \approx 0,436 \mathrm{MeV}=$ $\Gamma_{\nu}^{\uparrow}\left(\right.$ pois $\left.\Gamma_{\pi}^{\dagger} \approx 0\right)$. Ainda, levando em conta a correção de $\sim 2 \%$, devido ao canal $k=\alpha$, temos :

$$
\begin{aligned}
0,839 & \leq \mu \leq 0,859 \\
0,436 & \leq \Gamma^{\uparrow} \leq 0,499 \mathrm{MeV} .
\end{aligned}
$$

O cálculo de $\Gamma^{\uparrow}$ e de $\mu$, com a RPA no contínuo da sec.1.2, pode fornecer $\Gamma^{\uparrow}$ e $\mu$ no sentido das eqs.2.22 e 2.23 somente, já que levamos em conta 
apenas as excitações de $1 \mathrm{p}-1 \mathrm{~b}$ para prótons e neutrons, resultando em:

$$
\Gamma^{\dagger}(R P A) \approx 0,520 \mathrm{MeV} \Rightarrow \mu(R P A) \approx 0,812 .
$$

Comparando os resultados desta seção com aqueles da sec. 1.2, podemos afirmar que existe um bom acordo entre eles, relacionados com a largura $\Gamma^{\dagger}$ e o parâmetro $\mu$. Porém, para as larguras de escape parciais dos neutrons, existe um bom acordo apenas para os neutrons que populam o estado fundamental do $Z r^{89}$, havendo neste caso, também um bom acordo com a análise do trabalho experimental [Bo 89]. Divergências deste tipo, também ocorrem nas análises feitas por Bracco [Br 88], para as larguras parcias de escape da RGE0 do $P b^{208}$. 


\section{Capítulo 3}

\section{Conclusões}

A inclusão do contínuo em cálculos de RPA através das energias não perturbadas das ressonâncias de partícula-única [Pi 87, Te 91a], fornece resultados bastantes razoáveis para as larguras de escape $\Gamma^{\dagger}$ das RG's, e o importante nestes cálculos é que, se a ressonância for isolada, podemos obter a largura $\Gamma^{\dagger}$ como uma soma das larguras parciais das várias componentes de $1 \mathrm{p}-1 \mathrm{~b}$ que expandem a RG.

As análises dos espectros experimentais de prótons e neutrons, através do modelo híbrido com separação dos canais de partícula ( sec.2.1), mostram que os resultados explicam razoavelmente bem os dados experimentais, apresentando também, um bom acordo com os cálculos de RPA no contínuo ( sec.1.2). A grande vantagem nestas análises é que a separação dos canais simplifica muito os cálculos, fornecendo um parâmetro de mistura $\mu_{k}$ para cada tipo de partícula emitida, e com os possíveis $\mu_{k}$ 's obtemos o parâmetro $\mu$ total, possibilitando o cálculo das larguras de escape parciais e total para cada canal de partícula. A simplificação está no fato 
de podermos controlar os $\mu_{k}$ 's em cada espectro, e não no controle de um $\mu$ total para os vários tipos de partículas emitidas, o que permite extrair conclusões com maior transparência do que das análises com o modelo híbrido usual [Di 86b, $\mathrm{Br} 88$ ]. Tais vantagens são conseguidas às custas de um cálculo aproximado, mas nas condições em que estas aproximações são válidas, os resultados são muito satisfatórios.

Na tabela 3.1, apresentamos um resumo dos resultados obtidos nos cálculos dos caps.1 e 2 para o $Z r^{90}$, juntamente com os resultados do cálculo estatístico efetuado no trabalho experimental [Bo 89]. 
Tabela 3.1: Resultados obtidos dos cálculos das secs.1.2 (RPA no contínuo), $2.3 \mathrm{e}$ 2.4 (MH - modelo híbrido) para o $Z r^{90}$, em comparação com os cálculos estatísticos do trabalho experimental (Bo 89). Note-se que, em nossos cálculos, as contribuições de prótons e neutrons são normalizados à $P_{\pi}+P_{\nu}$ (eqs.2.22 e 2.23).

\begin{tabular}{|c|c|c|c|c|c|}
\hline & MH & RPA & Bo 89 \\
\hline$k$ & $i$ & $E_{x i}(M e V)$ & $\Gamma_{k i}^{\uparrow}(M e V)$ & $\Gamma_{k i}^{\uparrow}(M e V)$ & $\Gamma_{k i}^{\uparrow}(M e V)$ \\
\hline \multirow{3}{*}{$\nu$} & $9 / 2^{+}$ & 0,0 & 0,163 & 0,170 & 0,155 \\
\hline & $1 / 2^{-}$ & 0,59 & 0,082 & 0,0 & 0,0 \\
\hline & $3 / 2^{-}$ & 1,09 & 0,082 & 0,0 & 0,0 \\
\hline \multirow[t]{3}{*}{$Z r^{89}$} & $5 / 2^{-}$ & 1,45 & 0,109 & 0,330 & 0,0 \\
\hline & \multicolumn{2}{|c|}{$\Gamma_{\nu}^{\uparrow}(M e V)$} & 0,436 & 0,500 & 0,155 \\
\hline & \multicolumn{2}{|r|}{$\mu_{\nu}$} & 0,840 & 0,817 & - \\
\hline \multirow{2}{*}{$\begin{array}{c}\pi \\
Y^{89}\end{array}$} & \multicolumn{2}{|c|}{$\Gamma_{\pi}^{\uparrow}(\mathrm{MeV})$} & 0,0 & 0,020 & 0,0 \\
\hline & \multicolumn{2}{|r|}{$\mu_{\pi}$} & 1,0 & 0,935 & - \\
\hline \multirow[t]{2}{*}{$\nu+\pi$} & \multicolumn{2}{|r|}{$\Gamma^{\uparrow}$} & 0,436 & 0,520 & 0,155 \\
\hline & \multicolumn{2}{|r|}{$\mu$} & 0,839 & 0,812 & - \\
\hline
\end{tabular}




\section{Apêndice A}

\section{Transformação da matriz}

\section{complexa da RPA no contínuo em}

\section{sua equivalente real}

A diagonalização da matriz complexa das eqs. 1.41 envolve algumas dificuldades técnicas, devido ao fato de que a matriz A é uma matriz complexa (as energias de $1 \mathrm{p}-1 \mathrm{~b}$ não perturbadas $\hat{\varepsilon}_{p b}=\hat{\varepsilon}_{p}-\hat{\varepsilon}_{b}$ são complexas quando a partícula está no contínuo), fazendo com que a normalização dos vetores em 1.42 não seja muito bem satisfeita. Por exemplo, a diagonalização através da rotina EIGCC do pacote de rotinas IMSL, fornece a norma $\left\langle\tilde{R}_{n} \mid R_{n^{\prime}}\right\rangle$ com precisão da ordem de $10^{-1}$. Para contornarmos esta dificuldade técnica, desenvolvemos um método [Te 93b] para transformarmos a matriz complexa de 1.41 em sua equivalente matriz real, cuja diagonalização (através da rotina EIGRF da IMSL) apresenta soluções bem mais 
precisas $\left(\sim 10^{-5}\right)$ do que aquela da matriz original. Para fazer essa transformação, nos baseamos no trabalho de Chi [Ch 70], onde se transforma a matriz real $2 \mathrm{~N} \mathrm{x}$ $2 \mathrm{~N}$ de RPA, em um problema envolvendo a solução de uma matriz real de ordem N. Da mesma forma, vamos transformar a nossa matriz complexa $2 \mathrm{~N} \mathrm{x} 2 \mathrm{~N}$, em outra $\mathrm{N} \times \mathrm{N}$, e depois transformamos esta matriz complexa $\mathrm{N} \mathrm{x} \mathrm{N} \mathrm{em} \mathrm{sua} \mathrm{equivalente} \mathrm{real}$ $2 \mathrm{~N} \times 2 \mathrm{~N}$. A simplificação da solução está no fato de que em vez de diagonalizarmos uma matriz complexa $2 \mathrm{~N} \times 2 \mathrm{~N}$, vamos diagonalizar uma matriz real de mesma ordem. Primeiro transformamos a matriz complexa das eqs.1.41, através de uma transformação unitária com o operador J [Ch 70],

$$
\begin{aligned}
J & =\frac{1}{\sqrt{2}}\left(\begin{array}{cc}
I & I \\
-I & I
\end{array}\right), \\
J J^{\dagger} & =J^{\dagger} J=I,
\end{aligned}
$$

onde I é a identidade. Chamando de $M$ a matriz complexa das eqs.1.41:

$$
M=\left(\begin{array}{cc}
A & B \\
-B & -A
\end{array}\right),
$$


fazemos a transformação $J^{\dagger} M J$, e obtemos as seguintes equações :

$$
\begin{aligned}
(A+B) \beta & =\varepsilon \alpha \\
(A-B) \alpha & =\varepsilon \beta \\
\left(\begin{array}{c}
\alpha \\
\beta
\end{array}\right) & =J^{\dagger}\left(\begin{array}{l}
X \\
Y
\end{array}\right) .
\end{aligned}
$$

As equações acima, podem ser reescritas na seguinte forma :

$$
\begin{aligned}
& C \alpha=\varepsilon^{2} \alpha, \\
& D \beta=\varepsilon^{2} \beta,
\end{aligned}
$$

onde $\alpha$ e $\beta$ são dados em A.2, e as matrizes $C$ e $D$ são definidas como:

$$
\begin{aligned}
C & =(A+B)(A-B) \\
D & =(A-B)(A+B), \\
\left(\begin{array}{l}
\alpha \\
\beta
\end{array}\right) & =J^{\dagger}\left(\begin{array}{l}
X \\
Y
\end{array}\right) \\
\beta & =\varepsilon^{-1}(A-B) \alpha .
\end{aligned}
$$

Podemos então resolver a primeira das eqs. em A.3 para $\mathrm{C}$ e $\alpha$, e depois obter $\beta$ por A.4. Desta forma, vamos reescrever a equação que envolve a 
matriz $\mathrm{C}$, complexa $\mathrm{N} \times \mathrm{N}$, da seguinte forma :

$$
\begin{array}{r}
P^{\prime}=\left(\begin{array}{cc}
C & 0 \\
0 & C^{*}
\end{array}\right), \\
\left(\begin{array}{cc}
C & 0 \\
0 & C^{*}
\end{array}\right)\left(\begin{array}{l}
\alpha \\
\alpha_{c}
\end{array}\right)=\lambda\left(\begin{array}{l}
\alpha \\
\alpha_{c}
\end{array}\right),
\end{array}
$$

com $\lambda$ sendo igual a $\varepsilon^{2}$ ou $\varepsilon^{* 2}$, correspondendo às soluções $\alpha$ e $\alpha_{c}$, respectivamente. Agora, transformamos a matriz $P^{\prime}$ em uma matriz real $P$, através da transformação $P=J^{\prime} P^{\prime} J^{\prime \dagger}$, com $J^{\prime}$ sendo definido como :

$$
J^{\prime}=\frac{1}{\sqrt{2}}\left(\begin{array}{cc}
I & I \\
-i I & i I
\end{array}\right)
$$

com $J^{\prime} J^{\prime} \dagger=J^{\prime} \dagger J^{\prime}=I$, e obtemos as seguintes equações :

$$
P \gamma=\lambda \gamma,
$$

onde :

$$
P=\left(\begin{array}{cc}
\operatorname{Re}(C) & -\operatorname{Im}(C) \\
\operatorname{Im}(C) & \operatorname{Re}(C)
\end{array}\right)
$$




$$
\gamma=\frac{1}{\sqrt{2}}\left(\begin{array}{c}
\left(\alpha+\alpha_{c}\right) \\
-i\left(\alpha-\alpha_{c}\right)
\end{array}\right)
$$

cujas soluções envolvem a diagonalização da matriz real $P$. As soluções da eq.A.7, são:

$\lambda=\varepsilon^{2}, \operatorname{com} \alpha_{c}=0:$

$$
\gamma=\frac{1}{\sqrt{2}}\left(\begin{array}{c}
\alpha \\
-i \alpha
\end{array}\right)
$$

e $\lambda=\varepsilon^{*^{2}}, \operatorname{com} \alpha=0$ :

$$
\gamma=\frac{1}{\sqrt{2}}\left(\begin{array}{c}
\alpha_{c} \\
i \alpha_{c}
\end{array}\right)
$$

Das soluções da eq.A.7, juntamente com a eq.A.4, obtemos as soluções das eqs.1.41, as quais são dadas por :

$$
\begin{aligned}
\varepsilon & =\sqrt{\lambda} \\
\left(\begin{array}{l}
X \\
Y
\end{array}\right) & =\frac{1}{\sqrt{2}}\left(\begin{array}{c}
\alpha+\beta \\
-\alpha+\beta
\end{array}\right) .
\end{aligned}
$$


O método apresenta problemas para soluções $\varepsilon=0$, mas como essas soluções representam estados espúrios (para excitações do tipo dipolo $1^{-}$), elas são descartadas quando $\lambda=0$ em A.5. Estas transformações apresentam precisão maior do que $10^{-5}$ para a norma na eq.1.42. 


\section{Apêndice B}

\section{Densidade de níveis para os}

\section{núcleos $Y^{89}$ e $Z r^{89}$}

Neste apêndice vamos descrever o modelo utilizado no cálculo das densidades de níveis dos núcleos residuais $Y^{89}$ e $Z r^{89}$, resultantes do decaimento da RGE0 do $Z r^{90}$ por emissão de prótons e neutrons, respectivamente.

A hamiltoniana do sistema é descrita em termos do modelo partícula-vibrador [Di 82] :

$$
H=H_{s p}+H_{c o l}+H_{i n t}+H_{r e s},
$$

onde $H_{s p}$ e $H_{c o l}$ fornecem as energias de partícula -independente e do caroço, respectivamente, $H_{\text {int }}$ descreve as interações partícula-vibrador, e $H_{\text {res }}$ as interações entre as partículas fora do caroço, tratadas com a interação residual de MSDI.

O caroço utilizado é o $S r^{88}$, com as energias dos fônons $E_{c}\left(0^{+}\right)=$ 
Tabela B.1: Energias de partícula-independente para o $Y^{89}$ e $Z r^{89}$.

\begin{tabular}{|c|c|}
\hline \multicolumn{2}{|c|}{ próton } \\
\hline$j^{\pi}$ & $\varepsilon_{\pi}(\mathrm{MeV})$ \\
\hline $1 / 2^{-}$ & 0,00 \\
\hline $9 / 2^{+}$ & 0,91 \\
\hline \hline \multicolumn{2}{|c|}{ nêutron } \\
\hline$j^{\pi}$ & $\varepsilon_{\nu}(\mathrm{MeV})$ \\
\hline $9 / 2^{+}$ & 0,00 \\
\hline $1 / 2^{-}$ & 0,39 \\
\hline $3 / 2^{-}$ & 0,87 \\
\hline $5 / 2^{-}$ & 1,26 \\
\hline
\end{tabular}

$0,0 \mathrm{MeV}, E_{c}\left(2^{+}\right)=1,836 \mathrm{MeV}$ e $E_{c}\left(3^{-}\right)=2,734 \mathrm{MeV}$.

Para o $Z r^{89}$ acoplamos o $S r^{88}$ a dois prótons $\left(Y^{89}\right)$ e um buraco de nêutron ( $\left.Z r^{89}\right)$, com energias de partícula-independente dadas na tab.B.1 .

A densidade de níveis deste núcleo, foi obtida utilizando o fônon $0^{+}$, um e dois fônons $2^{+}$, e um fônon $3^{-}$do $S r^{88}$ acoplados aos níveis da tab.B.1. $\mathrm{Na}$ tab.B.2 apresentamos os resultados obtidos em comparação com os dados experimentais [NDS] até a energia $E_{x}\left(Z r^{89}\right)=2,0 \mathrm{MeV}$, onde podemos observar um razoável acordo entre o cálculo e os dados experimentais [NDS]. 
Tabela B.2: Níveis de energia e spin do $Z r^{89}$ obtidos teóricamente em comparação com os resultados experimentais.

\begin{tabular}{|c|c|c|}
\hline \multicolumn{3}{|c|}{$Z r^{89}(\mathrm{MeV})$} \\
\hline$j^{\pi}$ & EXP & TEO \\
\hline $9 / 2^{+}$ & 0,00 & 0,00 \\
\hline $1 / 2^{-}$ & 0,59 & 0,28 \\
\hline $3 / 2^{-}$ & 1,09 & 1,17 \\
\hline $11 / 2^{+}$ & - & 1,43 \\
\hline $5 / 2^{-}$ & 1,45 & 1,64 \\
\hline $9 / 2^{+}$ & 1,51 & 1,59 \\
\hline $5 / 2^{+}$ & 1,63 & 1,66 \\
\hline $7 / 2^{+}$ & - & 1,69 \\
\hline $3 / 2^{-}$ & 1,74 & 2,19 \\
\hline $5 / 2^{+}$ & 1,83 & 2,39 \\
\hline $3 / 2^{-}$ & 1,86 & 2,29 \\
\hline $13 / 2^{+}$ & 1,94 & 1,61 \\
\hline
\end{tabular}


Tabela B.3: Distribuição dos níveis de energia do $Z r^{89}$ previstos teóricamente. O total de níveis experimentais, conta os níveis com ou sem spins medidos. Acima de $2,0 \mathrm{MeV}$ existe uma indeterminação experimental muito grande quanto aos spins, e por isso colocamos apenas o número total de níveis.

\begin{tabular}{|c|c|c|c|c|c|c|c|c|c|c|c|c|}
\hline spin & \multicolumn{2}{|c|}{$1 / 2$} & \multicolumn{2}{|c|}{$3 / 2$} & \multicolumn{2}{|c|}{$5 / 2$} & \multicolumn{2}{|c|}{$7 / 2$} & \multicolumn{2}{|c|}{$\geq 9 / 2$} & \multicolumn{2}{|c|}{ total } \\
\hline$\Delta E_{x}(\mathrm{MeV})$ & teo & $\exp$ & teo & $\exp$ & teo & $\exp$ & teo & $\exp$ & teo & $\exp$ & teo & $\exp$ \\
\hline $0-1$ & 1 & 1 & 0 & 0 & 0 & 0 & 0 & 0 & 1 & 1 & 2 & 2 \\
\hline $1-2$ & 0 & 0 & 1 & 3 & 2 & 3 & 1 & 1 & 3 & 1 & 7 & 8 \\
\hline $2-3$ & 3 & - & 3 & - & 4 & - & 5 & - & 23 & - & 38 & 28 \\
\hline $3-4$ & 4 & - & 9 & - & 10 & - & 12 & - & 31 & - & 66 & 23 \\
\hline $4-5$ & 8 & - & 17 & - & 24 & - & 27 & - & 126 & - & 202 & 11 \\
\hline
\end{tabular}

O cálculo prevê 315 níveis de energia entre $E_{x}\left(Z r^{89}\right)=0,0$ e 5,0 MeV , estando em bom acordo com o número de níveis previsto pela densidade utilizada em Bo 89. Na tab.B.3 apresentamos a distribuição de spins dos estados do $Z r^{89}$ em intervalos de $1,0 \mathrm{MeV}$ de energia.

Os níveis de energia e spin do núcleo residual $Y^{89}$ foram obtidos acoplando um próton ao $S r^{88}$ sem a interação residual. Neste caso utilizamos os níveis experimentais do $S r^{88}$ até $E_{x}\left(S r^{88}\right)=6,0 \mathrm{MeV}$. A densidade de níveis obtida é apresentada na tab.B.4. O cálculo não perturbado fornece 160 níveis obtidos no 
Tabela B.4: Distribuição dos níveis de energia do $Y^{89}$ previstos teóricamente. O total de níveis experimentais, conta os níveis com ou sem spins medidos. Acima de 3,0 MeV existe uma indeterminação experimental muito grande quanto aos spins, e por isso colocamos apenas o número total de níveis.

\begin{tabular}{|c|c|c|c|c|c|c|c|c|c|c|c|c|}
\hline spin & \multicolumn{2}{|c|}{$1 / 2$} & \multicolumn{2}{|c|}{$3 / 2$} & \multicolumn{2}{|c|}{$5 / 2$} & \multicolumn{2}{|c|}{$7 / 2$} & \multicolumn{2}{|c|}{$\geq 9 / 2$} & \multicolumn{2}{|c|}{ total } \\
\hline$\Delta E_{x}(\mathrm{MeV})$ & teo & $\exp$ & teo & $\exp$ & teo & $\exp$ & teo & $\exp$ & teo & $\exp$ & teo & $\exp$ \\
\hline $0-1$ & 1 & 1 & 0 & 0 & 0 & 0 & 0 & 0 & 1 & 1 & 2 & 2 \\
\hline $1-2$ & 0 & 0 & 1 & 1 & 1 & 1 & 0 & 0 & 0 & 0 & 2 & 2 \\
\hline $2-3$ & 0 & 0 & 0 & 1 & 2 & 1 & 2 & 2 & 3 & 3 & 7 & 7 \\
\hline $3-4$ & 4 & - & 5 & - & 4 & - & 3 & - & 7 & - & 23 & 21 \\
\hline $4-5$ & 3 & - & 6 & - & 9 & - & 11 & - & 46 & - & 75 & 41 \\
\hline $5-6$ & 2 & - & 4 & - & 5 & - & 6 & - & 34 & - & 51 & 48 \\
\hline
\end{tabular}

intervalo de energia de $E_{x}\left(Y^{89}\right)=0,0-6,0 \mathrm{MeV}$, estando em bom acordo com a densidade utilizada em Bo 89. 


\section{Apêndice C}

\section{Decaimento estatístico e modelo}

\section{híbrido}

No cálculo estatístico [Ha 52], assume-se a independência entre formação e decaimento do núcleo composto (NC), exceto pelas leis de conservação de energia, momento angular e paridade; envolvendo dois estágios : primeiro a formação do NC, e depois o seu decaimento por emissão de algum tipo de partícula energeticamente permitida, deixando um núcleo residual numa energia de excitação $\left(U_{i}\right)$ correspondente à energia da partícula emitida $\left(\varepsilon_{p}\right)$ :

$$
U_{i}=E_{x}-E_{l}-\varepsilon_{p},
$$

onde $E_{l}$ é o limiar de emissão da partícula, e $E_{x}$ é a energia de excitação . A teoria trata a seção de choque como sendo uma média dentro de um intervalo de 
energia $\Delta E$ que contém várias ressonâncias, desprezando as interferências entre canais. Para cada momento angular e paridade $J^{\pi}$ da excitação, temos :

$$
\bar{\sigma}_{i}\left(E_{x}, J^{\pi}\right)=\bar{\sigma}_{\text {form }}\left(E_{x}, J^{\pi}\right) \eta_{i}
$$

com $\eta_{i}$ sendo a probabilidade de que o núcleo composto formado $\left(\bar{\sigma}_{f o r m}\left(E_{x}, J^{\pi}\right)\right)$ decaia emitindo uma partícula, que vai popular o i-ésimo nível de energia do núcleo residual. As probabilidades $\eta_{i}$ são definidas em termos dos coeficientes de transmissão $T_{i}$, os quais são obtidos resolvendo a equação de Schröedinger com um potencial ótico $(V+i W)$, cuja parte imaginária representa a absorção da partícula pelo núcleo :

$$
\eta_{i}=\frac{T_{i}}{\sum_{j} T_{j}}
$$

Reescrevendo a eq.C.2 em termos dos $T_{i}$ 's, temos :

$$
\bar{\sigma}_{i}\left(E_{x}, J^{\pi}\right)=\bar{\sigma}_{f o r m}\left(E_{x}, J^{\pi}\right) \frac{T_{i}}{\sum_{j} T_{j}},
$$

onde o indice $i(j)$ representa tanto o canal de partícula (próton, nêutron, ... ), quanto os canais de momento angular e spin da partícula emitida e do núcleo residual, e a soma em $j$ deve ser feita sobre todos os canais de saída possíveis. No modelo híbrido [Di 86b], utilizando a representação ótica de 
fundo de Kawai, Kerman e McVoy [Ka 73], escreve-se a seção de choque mediada em energia como uma soma devido aos processos semi-diretos $\left(\sigma_{i D}\right)$ e de NC $\left(\sigma_{i C}\right)$ :

$$
\bar{\sigma}_{i}\left(E_{x}, J^{\pi}\right)=\sigma_{i D}\left(E_{x}, J^{\pi}\right)+\sigma_{i C}\left(E_{x}, J^{\pi}\right) .
$$

Devido ao fato de que a partícula pode ser emitida, tanto pela RG, tratada como um "doorway", como pelo sistema composto, os coeficientes de transmissão $T_{i}$ 's, são decompostos em duas componentes :

$$
T_{i}=\tau_{i C}+\mu \tau_{i D}
$$

onde $\tau_{i C}$ é o coeficiente de transmissão devido a emissão da partícula pelo sistema composto, $\tau_{i D}$ é a contribuição devido à RG, e $\mu$ é um parâmetro que mede o grau de fragmentação da RG $\left(\mu=\Gamma^{\downarrow} / \Gamma\right.$ com $\left.\Gamma=\Gamma^{\uparrow}+\Gamma^{\downarrow}\right)$. A contribuição do sistema composto é, agora, normalizada à $\mu$, e para garantir a unitariedade da seção de choque, a contribuição semi-direta deve ser normalizada a $\Gamma^{\dagger} / \Gamma=1-\mu$ :

$$
\sigma_{i D}=\bar{\sigma}_{f o r m}(1-\mu) \frac{\tau_{i D}}{\sum_{j} \tau_{j D}}
$$

com $\tau_{i D}=2 \pi \rho_{D} \Gamma_{i D}\left(\Gamma^{\uparrow}=\sum_{i} \Gamma_{i D}\right)$, e $\rho_{D}$ é a densidade de excitações $1 \mathrm{p}-1 \mathrm{~b}$. Reescrevendo para os "branching ratios", temos :

$$
b_{i}=(1-\mu) \frac{\tau_{i D}}{\sum_{j} \tau_{j D}}+\mu \frac{\tau_{i C}+\mu \tau_{i D}}{\sum_{j}\left(\tau_{j C}+\mu \tau_{i D}\right)},
$$


o que nos permite analisar o decaimento das RG's levando em conta as contribuições semi-diretas e estatísticas, simultâneamente. 


\section{Referências}

[Ba 47] G.C.Baldwin and G.S.Klaiber - Phys.Rev.71(1947)3.

[Be 79] O.Bersilon - SCAT Optical Model Code, CEA Bruyeres-le-Chatel, France(1979).

[Bo 69] A.Bohr e B.R.Mottelson - Nuclear Structure V.I (Benjamin, New York, 1969).

[Bo 89] W.T.A.Borghols, S.Brandemburg, J.H.Meier, J.M.Schippers, M.M.Sharma, A.Van der Wood, M.N.Harakeh, A.Lindholm, L.Nilsson, S.Crona, A.Hakansson, L.P.Ekström, N.Olsson and R.de Leo Nucl.Phys.A504(1989)231.

[Br 59] G.Brown and M.Bolsterli - Phys.Rev.Lett.3(1959)472.

[Br 79] G.Brown, J.S.Dehesa and J.Speth - Nucl.Phys.A330(1979)290.

[Br 87] S.Brandenburg, W.T.A.Borghols, A.G.Drentje, L.P.Ekström, M.N.Harakeh and A.Van der Woude - Nucl.Phys.A466(1987)29. 
[Br 88] A.Bracco, J.R.Beene, N.Van Giai, P.F.Bortignon, F.Zardi and R.A.Broglia - Phys.Rev.Lett.60(1988)2603.

[Br 89] S.Brandenburg, W.T.A.Borghols, A.G.Drentje, A.Van der Woude, M.N.Harakeh, L.P.Ekström, A.Hakanson, L.Nilsson, N.Olsson and R.de Leo - Phys.Rev.C39(1989)2448.

[Bu 67] B.Buck and A.D.Hill - Nucl.Phys.A95(1967)271.

[Ca 69] J.R.Calarco - Ph.D. Thesis University of Illinois (1969).

[Ch 70] B.Chi - Nucl.Phys.A146(1970)449.

[Ch 87] Ph.Chomaz, N.Van Giai and S.Stringari - Phys.Lett.B189(1987)375.

[Co 85] G.Co and S.Krewald - Nucl.Phys.A433(1985)392.

[Cu 89] P.Curutchet, T.Vertse and R.J.Liotta - Phys.Rev.C39(1989)1020.

[De 77] J.S.Dehesa, S.Krewald, J.Speth and A.Faessler - Phys.Rev.C15(1977)1858.

[Di 73] W.Dilg, W.Schantl, H.Vonach and M.Uhl - Nucl.Phys.A217(1973)269.

[Di 82] H.Dias and F.Krmpotic - Phys.Rev.C25(1982)2059.

[Di 84] H.Dias and E.Wolynec - Phys.Rev.C30(1984)1164.

[Di 86a] H.Dias, N.Teruya and E.Wolynec - Phys.Rev.C33(1986)1955.

[Di 86b] H.Dias, M.S.Hussein and S.K.Adhikari - Phys.Rev.Lett.57(1986)1998. 
[Ey 84] W.Eyrich, K.Fuchs, A.Hoffmann, U.Sheib, H.Steuer and H.Rebel Phys.Rev.C29(1984)418.

[Fo 91] G.Foglia, Tese de Doutorado - IFUSP 1991.

[Gi 87] N.Van Giai, P.F.Bortignon, F.Zardi and R.A.Broglia Phys.Lett.B199(1987)155.

[Go 48] M.Goldhaber and E.Teller - Phys.Rev.74(1948)1046.

[Ha 52] W.Hauser and H.Feshbach - Phys.Rev.87(1952)366.

[Ha 77] M.N.Harakeh, K.Van der Borg, T.Ishimatsu, H.P.Morsch, A.Van der Woude and F.E.Bertrand - Phys.Rev.Lett.38(1977)676.

[Ha 82] R.de Haro, S.Krewald and J.Speth - Nucl.Phys.A388(1982)265.

[Ka 73] M.Kawai, A.Kerman and K.McVoy - Ann.Phys.75(1973)156.

[Le 72] M.B.Lewis and F.E.Bertrand - Nucl.Phys.A196(1972)337.

[Li 76] K.F.Liu and N.Van Giai - Phys.Lett.B65(1976)23.

[NDS] Nuclear Data Sheets :

H.W.Müller - 54(1988)39,

H.Sievers - 58(1.989)400.

[Pi 71a] R.P.Pithan and T.H.Walcher - Phys.Lett.B36(1971)563. 
[Pi 71b] A.F.R.de Toledo Piza - Technical Report, Universidade de São Paulo (1971), não publicado.

[Pi 87] A.F.R.de Toledo Piza - Rev.Bras.Fís.17(1987)195.

[Pi 89] A.F.R.de Toledo Piza and G.A.Foglia - Proc.Int.Nucl.Phys.Conf. V-2(1989)453 (São Paulo - Brasil).

[Ra 79] J.Rapaport, V.Kulkarni and R.W.Finlay - Nucl.Phys.A330(1979)15.

[Ri 78] G.A.Rinker and J.Speth - Nucl.Phys.A306(1978)360.

[Ro 70] D.J.Rowe - Nuclear Collective Motion - Methuen and Co. LTD. (London-1970).

[Sa 90] H.Sagawa and M.Honma - Phys.Lett.B251(1990)17.

[Sc 84] B.Schwesinger and J.Wambach - Nucl.Phys.A426(1984)253.

[Se 86] F.Ajzemberg-Selove - Nucl.Phys.A460(1986)1.

[Sh 75] S.Shlomo and G.Bertsch - Nucl.Phys.A243(1975)507.

[St 50] V.H.Steinwedel and J.H.D.Jensen - Z.Naturf.5a(1950)413.

[St 81] H.Steuer, W.Eyrich, A.Hofmann, H.Ortner, U.Scheib, R.Stamminger and D.Steuer - Phys.Rev.Lett.47(1981)1702.

[Ta 90] Y.Takahashi and S.Yoshida - Nucl.Phys.A507(1990)371.

[Te 88] N.Teruya, H.Dias and E.Wolynec - Phys.Rev.C37(1988)2121. 
[Te 89] N.Teruya, E.Wolynec and H.Dias - Rev.Bras.Fís.19(1989)263.

[Te 91a] N.Teruya, A.F.R.de Toledo Piza and H.Dias - Phys.Rev.C44(1991)537.

[Te 91b] N.Teruya, C.A.Bertulani, S.Krewald, H.Dias and M.S.Hussein Phys.Rev.C43(1991)R2049.

[Te 93a] N.Teruya, H.Dias and A.F.R.de Toledo Piza - Nucl.Phys.A556(1993)157.

[Te 93b] N.Teruya, M.Kyotoku and H.Dias - Int.Jour.Mod.Phys.E2(1993)265.

[Te 93c] N.Teruya and H.Dias - submetido ao Phys.Rev.C.

[Te 93d] N.Teruya and H.Dias - em preparação .

[Yo 83] S.Yoshida - Suppl.of the Prog.of Theor.Phys.74-75(1983)142.

[Wa 70] W.L.Wang and C.M.Shakin - Phys.Lett.B32(1970)421.

[Wo 87] A.Van der Woude - Prog.Part.Nucl.Phys.18(1987)217. 